\title{
Optical Caustics Observed in Light Scattered by an Oblate Spheroid
}

James A. Lock

Cleveland State University, j.lock@csuohio.edu

Feng Xu

Follow this and additional works at: https://engagedscholarship.csuohio.edu/sciphysics_facpub

Part of the Physics Commons

How does access to this work benefit you? Let us know!

\section{Publisher's Statement}

This paper was published in Applied Optics and is made available as an electronic reprint with the permission of OSA. The paper can be found at the following URL on the OSA website: http://www.opticsinfobase.org/ao/abstract.cfm?URI=ao-49-8-1288. Systematic or multiple reproduction or distribution to multiple locations via electronic or other means is prohibited and is subject to penalties under law.

\section{Original Citation}

Lock, James A. and Feng Xu. "Optical Caustics Observed in Light Scattered by an Oblate Spheroid." Applied Optics 49 (2010): 1288-1304.

\section{Repository Citation}

Lock, James A. and Xu, Feng, "Optical Caustics Observed in Light Scattered by an Oblate Spheroid" (2010). Physics Faculty Publications. 73.

https://engagedscholarship.csuohio.edu/sciphysics_facpub/73

This Article is brought to you for free and open access by the Physics Department at EngagedScholarship@CSU. It has been accepted for inclusion in Physics Faculty Publications by an authorized administrator of EngagedScholarship@CSU. For more information, please contact library.es@csuohio.edu. 


\title{
Optical caustics observed in light scattered by an oblate spheroid
}

\author{
James A. Lock ${ }^{1,3}$ and Feng $\mathrm{Xu}^{2,4}$ \\ 'Department of Physics, Cleveland State University, Cleveland, Ohio 44115, USA \\ ${ }^{2}$ Fachgebiet Strömungslehre und Aerodynamik, Technische Universität Darmstadt, \\ Petersenstrasse, 30, 64289 Darmstadt, Germany \\ 3j.lock@csuohio.edu \\ 4f3_xu@yahoo.com
}

Received 13 November 2009; revised 27 January 2010; accepted 29 January 2010; posted 29 January 2010 (Doc. ID 119690); published 4 March 2010

\begin{abstract}
The electromagnetic fields scattered when a plane wave is incident on an oblate spheroid in the side-on orientation may be calculated using a generalization of Mie theory, and the results may be decomposed in a Debye series expansion. A number of optical caustics are observed in the computed scattered intensity for the one internal reflection portion of the Debye series for scattering angles in the vicinity of the firstorder rainbow, and are analyzed in terms of the rainbow, transverse cusp, and hyperbolic umbilic caustics of catastrophe optics. The specific features of these three caustics are described, as is their assembly into the global structure of the observed caustics for spheroid scattering. It is found that, for a spheroid whose radius is an order of magnitude larger than the wavelength of the incident light, the interference structure accompanying the transverse cusp and hyperbolic umbilic caustics is only partially formed. () 2010 Optical Society of America
\end{abstract}

OCIS codes: $\quad 260.1960,290.4020$.

\section{Introduction}

When a plane wave is incident on an oblate spheroid in the side-on orientation, the scattered intensity observed on a distant viewing screen exhibits a number of prominent and interesting optical caustics. Although these caustic structures were experimentally observed [1] and successfully described in terms of approximate theoretical models [2-9] a number of years ago, to the best of our knowle dge they have never been examined using exact electromagnetic theory (see, however, Fig. 1c of [10]), such as the extension of Mie theory to scattering by a homogeneous spheroid [11-14]. The purpose of this paper is to identify and interpret these caustic structures in the numerical solution of the exact electromagnetic equations when the spheroid's radius is about 12 times the wavelength of the incident light.

0003-6935/10/081288-17\$15.00/0

(C) 2010 Optical Society of America
The interpretation of the structures observed in the calculated scattered intensity requires an understanding of the properties of a number of optical caustics. These scattering caustics and their associated diffraction patterns possess two levels of structure, an overall or global structure, and a local structure in the vicinity of the global caustic's point of greatest focusing $[15,16]$. It is well known that the rainbow, the simplest of all the caustics, is incompletely formed for scattering by a small size parameter sphere. The fine details of the rainbow's interference structure become fully resolved only when the sphere size parameter becomes very large [17]. Similarly, it is of interest to determine which properties of the more complicated caustics associated with spheroid scattering are resolved when the spheroid radius is only an order of magnitude larger than the incident wavelength.

The electromagnetic fields scattered by a spheroid can be decomposed into an infinite series of terms known as the Debye series, where the individual 
contributions to wave scattering corresponding to diffraction-plus-reflection, transmission, and transmission following $p-1$ internal reflections can be isolated and studied individually [18]. Each scattering caustic is associated with a single Debye process. If the total scattered intensity were calculated, the appearance of the caustics associated with a single Debye process would be masked or obscured by light scattered at the same angle by all the other processes, and by its interference with the specific process being studied. To eliminate these masking effects, the calculations reported here consider only the $p=2$ Debye process of transmission following one internal reflection. Our results are, thus, the small size parameter wave scattering analog of Figs. 2a-2e of [9], which were obtained using ray optics. The caustics examined here would be more completely formed if the scattered intensity could be calculated exactly for a much larger spheroid. But it is not at present feasible to do so because of difficulties encountered when the radial and angular spheroidal functions are computed for large spheroids having a large vertical-to-horizontal axis ratio [19]. But of even greater concern are the seemingly unavoidable numerical instabilities encountered due to the ill-conditioned nature of the large system of simultaneous linear equations resulting from the boundary condition matching that must be solved for the scattered field.

The body of this paper is organized as follows. In Section 2 we describe the scattering geometry and define the notation used in the remainder of this paper. In Sections $\underline{3}-\underline{5}$ we recount, extend, and physically interpret the formulas for the various properties of the rainbow, transverse cusp, and hyperbolic umbilic caustics using catastrophe optics methods applied to scattering by an oblate spheroid. The methods of catastrophe optics quantitatively describe the local structure of what are called structurally stable optical caustics, and can sometimes provide information concerning the global caustic structure, as well $[15,16]$. In Section 6 we present our numerical results for $p=2$ scattering by a spheroid whose size parameter is $2 \pi a / \lambda \approx 73$, and interpret the features observed in the scattered intensity in the context of the theoretical development of Secs. 35 . Transverse cusp caustics have also been observed [20-22] for scattering of a plane wave by an oblate spheroid with $p \geq 3$. In addition, a more complicated caustic progression has been predicted and observed $[9,23,24]$ at larger spheroid radius ratios for $p=2$ scattering. These higher $p=2$ caustics and the caustics for $p \geq 3$ are not examined in this paper.

\section{Scattering Geometry}

To motivate the scattering geometry, we first consider a sphere of radius $a$ and real refractive index $n$ whose center is at the origin of an $x, y, z$ rectangular coordinate system. A collection of parallel light rays of wavelength $\lambda$ and wave number $k=2 \pi / \lambda$ traveling in the positive $z$ direction is incident on the sphere. In this section, we are primarily interested in transverse electric (TE) polarized rays incident on the sphere in the horizontal $z x$ plane and whose electric field vector points in the vertical $y$ direction, as is illustrated in Fig. 1. We choose one of the incident rays to be the central ray and follow its path through the sphere to the scattering far zone. The central ray makes an angle $\theta_{i}{ }^{0}$ with respect to the normal to the sphere surface. Its deflection angle after undergoing $p-1$ internal reflections within the sphere before exiting is

$$
\Theta_{0}=(p-1) \pi+2 \theta_{i}{ }^{0}-2 p \theta_{t}{ }^{0},
$$

where $\theta_{t}^{0}$ is the angle between the central ray inside the sphere and the normal to the sphere surface, and

$$
\sin \left(\theta_{i}{ }^{0}\right)=n \sin \left(\theta_{t}{ }^{0}\right)
$$

The angle $\theta_{i}{ }^{0}$ is positive if the central ray is incident on the sphere with $x>0$, and the deflection angle $\Theta_{0}$ is positive when measured clockwise from the positive $z$ axis. A viewing screen is placed in the scattering far zone perpendicular to the $\Theta_{0}$ ray, and the origin of the viewing screen coordinate system is taken to be the point where the $\Theta_{0}$ ray intersects the screen. We wish to determine the scattered field in the vicinity of the origin of the viewing screen. Other incoming rays near the central ray have the angle of incidence

$$
\theta_{i}=\theta_{i}{ }^{0}+\varepsilon,
$$

and the deflection angle

$$
\Theta=\Theta_{0}+\Delta
$$

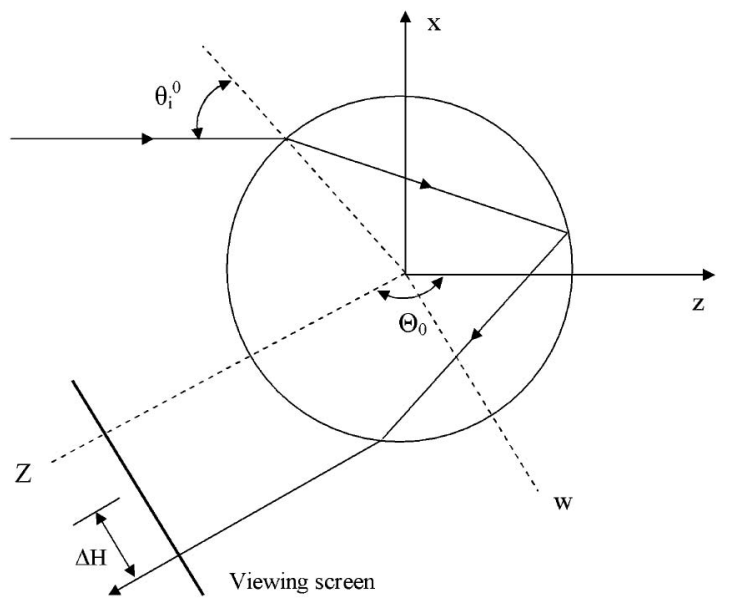

Fig. 1. Geometry for the physical optics model for a plane wave scattered by a sphere or by an oblate spheroid. The central incident ray makes an angle $\theta_{i}{ }^{\circ}$ with the normal to the spheroid surface in the horizontal $z x$ plane. The central ray's scattering angle is $\Theta_{o}$. The $w v$ plane, with $w$ horizontal and $v$ vertical, is the spheroid's exit plane. A far-zone viewing screen is normal to the $Z$ axis and has its origin at the point where it intersects the exiting central ray. The angular position of a point on the exit plane with respect to this origin is $\Delta_{H}$ in the horizontal direction and $\Delta_{V}$ in the vertical direction. 
where

$$
\begin{gathered}
\Theta=(p-1) \pi+2 \theta_{i}-2 p \theta_{t}, \\
\sin \left(\theta_{i}\right)=n \sin \left(\theta_{t}\right) .
\end{gathered}
$$

When $\Delta$ is positive, the deflection angle of the ray in question is larger than that of the central ray. Thus, for $p=2$ scattering in the vicinity of the rainbow, rays with positive $\Delta$ are closer to the backscattering direction than is the central ray.

We also consider a second set of rectangular coordinates rotated clockwise in the $z x$ plane with respect to the original coordinate system by the angle $\Theta_{0}$. The rotated $x, y$, and $z$ axes will hereafter be called $w, v$, and $Z$, respectively, and are also shown in Fig. 1 . The $w Z$ plane is horizontal and the $v$ axis is vertical. When calculating the optical path length of various rays through the sphere, a number of previous authors [25-27] have taken the sphere's entrance plane to be tangent to it at $z=-a$, and the exit plane tangent to it at $Z=a$. In this paper, we use another choice $[28,29]$ for the entrance and exit planes for reasons that will become apparent when we consider the transverse cusp and hyperbolic umbilic caustics in Sections 3 and $\underline{4}$. Specifically, after calculating the optical path length of a ray in the $z x$ plane from $z=$ $-a$ to $Z=a$, we proceed to move the entrance plane forward to $z=0$ and move the exit plane back to $Z=0$, as if the sphere were absent. Thus, for example, if the optical path length of the central ray were $2 a\left[1+n p \cos \left(\theta_{t}^{0}\right)-\cos \left(\theta_{i}{ }^{0}\right)\right]$ from $z=-a$ to $Z=a$, it would be $2 a\left[n p \cos \left(\theta_{t}^{0}\right)-\cos \left(\theta_{i}^{0}\right)\right]$ from $z=0$ to $Z=0$. We hereafter call $\Phi(0)$ the phase associated with the optical path length of the central ray. The shape of the outgoing wavefront as a function of $w$ and $v$ near the origin of the sphere's exit plane is $\Phi(w, v)$. Since $w$ and $v$ will be considered small, the outgoing wavefront is Taylor series expanded in powers of $w$ and $v$.

In the physical optics model of wave scattering, the outgoing wavefront in the exit plane centered on the deflection angle $\Theta_{0}$ is Fraunhofer diffracted a large distance $Z$ to give the scattered field as a function of $\Delta_{H}$ and $\Delta_{V}$, the horizontal and vertical angular displacements, respectively, with respect to $\Theta_{0}$ on the far-zone viewing screen. As is seen in Fig. 1 , a positive angle $\Delta_{H}$ on the viewing screen corresponds to the negative $w$ direction on the exit plane, and a positive angle $\Delta_{V}$ corresponds to the positive $v$ direction. The physical optics model scattered field is [30]

$$
\begin{aligned}
E\left(\Delta_{H}, \Delta_{V}\right)= & (-i k / 2 \pi Z) \exp (i k Z) t_{21}{ }^{0}\left(r_{11}{ }^{0}\right)^{p-1} t_{12}{ }^{0} \\
& \times\left(Q_{H} Q_{V}\right)^{1 / 2} \int_{-\infty}^{\infty} \mathrm{d} w \int_{-\infty}^{\infty} \mathrm{d} v \exp \{i[\Phi(w, v) \\
& \left.\left.+k w \Delta_{H}-k v \Delta_{V}\right]\right\}
\end{aligned}
$$

where $t_{21}{ }^{0}, r_{11}{ }^{0}$, and $t_{12}{ }^{0}$ are the TE-polarized Fresnel coefficients evaluated at $\theta_{i}{ }^{0}$ for transmission from the air (medium 2) into the sphere (medium 1), internal reflection within the sphere, and transmission back out, respectively. The factors $Q_{H}$ and $Q_{V}$ are the ray density amplification factors in the horizontal and vertical directions from the sphere's entrance plane to its exit plane. They describe the fanning out or constriction of a ray tube formed around the central ray as the ray propagates from the entrance plane to the exit plane [31]. The infinite limits of integration in Eq. (7) neglect the physical constraint that, in practice, the phase front is cut off by a finite effective aperture. In general, these omitted regions correspond to rapidly destructive interference, which produces only a negligible contribution to the integral.

\section{Rainbow Caustic}

The rainbow caustic, also known as the Airy caustic or fold caustic in the terminology of catastrophe optics, is a two-ray-to-zero-ray transition [15]. For scattering by a sphere following $p-1$ internal reflections, the Taylor series expansion of the outgoing wavefront in the exit plane for $\Theta_{0}$ in the vicinity of this caustic is

$$
\begin{aligned}
\Phi(w, v)= & \Phi(0)+k w^{2} / 2 R a-k h w^{3} / 3 a^{2} \\
& +k v^{2} / 2 S a+\ldots,
\end{aligned}
$$

where $R, h$, and $S$ are dimensionless, and $h$ and $S$ are assumed to be positive. The quantities $R$ and $S$ are the radii of curvature of the wavefront at the origin of the exit plane in the $w$ and $v$ directions, respectively, in units of $a$. A ray-tracing analysis for this geometry gives

$$
\begin{gathered}
R=-\cos \left(\theta_{i}{ }^{0}\right) /\left[2\left(1-p A_{0}\right)\right], \\
h=\left[\sin \left(\theta_{i}^{0}\right) / \cos ^{3}\left(\theta_{i}^{0}\right)\right] \\
\times\left[\left(1-p A_{0}{ }^{3}\right)-4\left(1-p A_{0}\right)^{3}\right], \\
S=-\cos \left(\Theta_{0}\right) \sin \left(\theta_{i}{ }^{0}\right) / \sin \left(\Theta_{0}\right), \\
Q_{H}=1, \\
Q_{V}=\left|1 / \cos \left(\Theta_{0}\right)\right|,
\end{gathered}
$$

where

$$
A_{0}=\cos \left(\theta_{i}^{0}\right) /\left[n \cos \left(\theta_{t}^{0}\right)\right] .
$$

The quantities $R, h$, and $S$ are functions of the choice of central ray $\theta_{i}{ }^{0}$. If the central ray is taken to be the Descartes rainbow ray (hereafter denoted by $r$ ) for $p \geq 2$, one has [32] 


$$
\begin{gathered}
\cos \left(\theta_{i}{ }^{r}\right)=\left[\left(n^{2}-1\right) /\left(p^{2}-1\right)\right]^{1 / 2}, \\
A_{r}=1 / p \\
R \rightarrow \infty, \\
h_{r}=\left(p^{2}-1\right) \sin \left(\theta_{i}^{r}\right) /\left[p^{2} \cos ^{3}\left(\theta_{i}^{r}\right)\right] \\
=\left(p^{2}-1\right)^{2}\left(p^{2}-n^{2}\right)^{1 / 2} /\left[p^{2}\left(n^{2}-1\right)^{3 / 2}\right] .
\end{gathered}
$$

With this choice of central ray, the smaller impact parameter supernumerary rainbow ray with $\varepsilon<0$ crosses the exit plane [33] at a negative value of $w$ and has a longer path length, while the larger impact parameter supernumerary ray with $\varepsilon>0$ crosses the exit plane at a positive value of $w$ and has a shorter path length. Thus, with our convention of $h$ being positive, a minus sign precedes it in Eq. (8). If one were interested in the scattered field on the viewing screen due to a single ray, the wavefront of Eq. ( would again be substituted into Eq. (7), but without the term proportional to $w^{3}$. Since $\bar{\Theta}_{0}$ is assumed here to be in the rainbow supernumerary region where two rays contribute at each scattering angle, the presence of the $w^{3}$ term insures that two rays participate, and smoothly joins the two locally parabolic wavefronts in the exit plane centered on the individual supernumerary rays. When $\Theta_{0}=\Theta_{r}$, the positive and negative horizontal curvatures of the wavefronts of the two supernumerary rays in the exit plane are effectively averaged to produce zero curvature, i.e., the $R \rightarrow \infty$ condition of Eq. (17). The increased focusing caused by this locally flat exiting wavefront in the horizontal direction produces the main peak in the rainbow intensity in the vicinity of $\Theta_{r}$.

In the short wavelength limit, the rainbow caustic in the $z x$ plane extends from the sphere surface, through the scattering near zone to the far zone, and is given by

$$
\begin{aligned}
z_{r} / a= & \sin \left(\Theta_{0}\right) \sin \left(\theta_{i}{ }^{0}\right) \\
& +\left\{\cos \left(\Theta_{0}\right) \cos \left(\theta_{i}{ }^{0}\right) /\left[2\left(1-p A_{0}\right)\right]\right\}, \\
x_{r} / a= & \cos \left(\Theta_{0}\right) \sin \left(\theta_{i}{ }^{0}\right) \\
& -\left\{\sin \left(\Theta_{0}\right) \cos \left(\theta_{i}{ }^{0}\right) /\left[2\left(1-p A_{0}\right)\right]\right\} .
\end{aligned}
$$

If $n=4 / 3$ and $0 \leq \theta_{i}{ }^{0}<\theta_{i}{ }^{r}$, the quantity $1-p A_{0}$ is negative, the rainbow caustic is virtual, and it extends from the sphere surface at $(z, x)=(a, 0)$ toward the asymptote [33] at the far-zone rainbow scattering angle. For this $\overline{\theta_{i}}$ interval, the horizontal radius of curvature $R$ is positive, corresponding to rays intersecting the exit plane in the horizontal direction as they diverge from an effective point source on the virtual caustic. For $\theta_{i}^{r}<\theta_{i}{ }^{0} \leq \pi / 2$, the quantity $1-p A_{0}$ is positive, the rainbow caustic is real, and it extends from the opposite end of the asymptote at the farzone rainbow scattering angle to the sphere surface at $(z, x)=\left[a \cos \left(\Theta_{0}-\theta_{i}{ }^{0}\right),-a \sin \left(\Theta_{0}-\theta_{i}{ }^{0}\right)\right]$. For this $\theta_{i}{ }^{0}$ interval, the horizontal radius of curvature $R$ is now negative, corresponding to rays intersecting the exit plane in the horizontal direction as they converge toward an effective point source on the real caustic. There is also a virtual axial spike caustic [34] formed by the backprojection of the rays in all planes of incidence with the same deflection angle $\Theta_{0}$. These projections all cross the $z$ axis at

$$
z=a \sin \left(\theta_{i}{ }^{0}\right) / \sin \left(\Theta_{0}\right) .
$$

The positive vertical radius of curvature $S$ in Eq. (8) in the vicinity of the rainbow corresponds to rays intersecting the exit plane in the vertical direction as they diverge from an effective point source on the virtual axial spike caustic.

Substituting Eqs. (8) and (17) into Eq. (7) with the choice of central ray $\bar{\Theta}_{0}=\Theta_{r}$, and not taking into account the slow variation of the Fresnel coefficients and the wavefront amplitude across the exit plane, the scattered field on the viewing screen in the vicinity of the Descartes rainbow angle for $\Delta_{V}=0$ and $\Delta_{H}$ small is

$$
\begin{aligned}
E\left(\Delta_{H}, 0\right)= & \exp \{i[k Z+\Phi(0)-\pi / 4]\}\left\{(k a)^{7 / 6}(2 \pi)^{1 / 2}\right. \\
& \left./\left[(k Z) h_{r}{ }^{1 / 3}\right]\right\}\left[\sin \left(\theta_{i}{ }^{r}\right) / \sin \left(\Theta_{r}\right)\right]^{1 / 2} \\
& \times t_{21}{ }^{r}\left(r_{11}{ }^{r}\right)^{p-1} t_{12}{ }^{r} \\
& \times A i\left[-(k a)^{2 / 3} \Delta_{H} / h_{r}{ }^{1 / 3}\right] .
\end{aligned}
$$

The Airy function $A i(X)$ is defined by the diffraction integral [35]

$$
A i(-X)=\left(3^{1 / 3} / 2 \pi\right) \int_{-\infty}^{\infty} \mathrm{d} w \exp \left\{i\left[w^{3}-(3)^{1 / 3} X w\right]\right\}
$$

Using the stationary phase approximation, the Airy function for $X$, evaluated not too close to the caustic position $X=0$ in the short wavelength limit, is [36]

$$
\begin{aligned}
& A i(-X) \approx 1 /\left(\pi^{1 / 2} X^{1 / 4}\right) \cos \left(2 X^{3 / 2} / 3-\pi / 4\right) \quad \text { for } X>0 \\
& \approx 1 /\left(2 \pi^{1 / 2}|X|^{1 / 4}\right) \exp \left(-2|X|^{3 / 2} / 3\right) \quad \text { for } X<0 .
\end{aligned}
$$

The $X>0$ region describes the supernumerary interference pattern and the $X<0$ region describes the faster-than-exponential falloff of the contribution of the complex ray. For $p=2$ scattering, the supernumerary region occurs for positive $\Delta_{H}$ corresponding to $\Theta>\Theta_{r}$. 


\section{Transverse Cusp Caustic}

The cusp caustic of catastrophe optics is a three-rayto-one-ray transition [15] whose canonical form is given by the diffraction integral [37]

$$
P(X, Y)=\int_{-\infty}^{\infty} \mathrm{d} w \exp \left[i\left(w^{4}+X w^{2}+Y w\right)\right]
$$

The properties of this integral have been studied by Pearcey [37] and by Connor and Farrelly [38]. In the short wavelength limit, the equation of the cusp is

$$
-(2 X / 3)^{3}=Y^{2} .
$$

The cusp point is at $(X, Y)=(0,0)$ and the cusp opens toward negative values of $X$. A stationary phase evaluation of Eq. (24) for $Y=0$ and $X$ evaluated not too close to the cusp point gives [38]

$$
\begin{aligned}
& P(X, 0) \approx(\pi / X)^{1 / 2} \exp (i \pi / 4) \quad \text { for } X>0 \\
& \quad \approx(\pi /|X|)^{1 / 2} \exp (-i \pi / 4)\left[1+i(2)^{1 / 2} \exp \left(-i X^{2} / 4\right)\right]
\end{aligned}
$$

for $X<0$.

The falloff of the Pearcey function along the $X$ axis for $X>0$ in Eq. (26) is much slower than that of the Airy function, and is the cause of the long cusp tails seen in photographs of the caustic [39].

For the remainder of this paper, we are concerned with $p=2$ scattering of a plane wave by an oblate spheroid with side-on incidence. The spheroid has radius $a$ in the horizontal $z x$ plane and radius $b<a$ along the vertical $y$ axis. For scattering with side-on incidence when $a / b$ is greater than some threshold value, a pair of transverse cusp caustics is experimentally observed [1] on a distant viewing screen. The cusps are centered on the horizontal axis between the backscattering direction and each of the locations where the $p=2$ rainbow crosses the horizontal axis. The cusps point toward the rainbows (see Fig. 2b of [9]). Each transverse cusp, together with its nearby rainbow caustic that becomes increasingly curved for spheroid scattering, form a hyperbolic umbilic caustic [15] that will be discussed in Section 5 . As $a / b$ increases, the cusps approach the rainbow caustics, then touch them, producing the focal section of the hyperbolic umbilic caustic (hereafter called hufs) and, finally, retract away from the rainbows (see Figs. 2c, 2d of [9]). The reason the rainbow becomes increasingly curved on the viewing screen in the local region centered on the horizontal axis is connected with the global structure of the caustic. Rays incident on the spheroid in the horizontal plane continue to reflect and refract from the circular cross section of radius $a$ and, thus, have the same Descartes rainbow angle as for sphere scattering. But rays incident on the spheroid in the vertical direction reflect and refract from an elliptical cross section, where the rainbow scattering angle increases toward $180^{\circ}$ as a function of $a / b$, in accordance with the Mobius prediction [40,41]. For unpolarized light and for $a / b$ less than the hufs condition to be given below in Eq. (34), the global rainbow caustic observed on the viewing screen that had been circular for sphere scattering now becomes increasingly flattened in the vertical direction for spheroid scattering. This flattening of the global rainbow caustic increases the localized curvature of the rainbow as it crosses the horizontal axis (see Fig. $2 b$ of [9]). The situation becomes more complicated, however, if either the incident light is unpolarized or the scattered light passes through a polarizing filter held by the observer. In this case, the TE-polarized portion of the main peak of the flattened global rainbow caustic is observed as bright, while the TMpolarized portion of the main peak is dim and is angularly shifted with respect to the TE portion [42]. The relative dimness of the TM portion of the peak is a consequence of the fact that, for the refractive index of water, the angle $\theta_{t}^{r}$ is quite close to the Brewster angle, greatly suppressing the TM internal reflection Fresnel coefficient.

When $\Delta_{V}=0$ on the viewing screen and the horizontal radius of curvature of the outgoing wavefront of the central ray is negative, the three rays participating in the transverse cusp caustic are the larger impact parameter rainbow supernumerary ray confined to the $z x$ plane and two skew rays. The skew rays enter the spheroid at equal distances $y_{i}{ }^{0}$ above and below the $z x$ plane, internally reflect at the spheroid equator in the $z x$ plane, exit the spheroid parallel to the $z x$ plane with identical path lengths, and interfere with the larger impact parameter supernumerary ray on the viewing screen. On the other hand, when the horizontal radius of curvature of the outgoing wavefront of the central ray is positive, the first participating ray now becomes the smaller impact parameter supernumerary ray. In each case, the other supernumerary ray is present, as well, but remains a spectator and does not participate in the formation of the transverse cusp caustic.

When the horizontal radius of curvature is negative corresponding to $a / b<(a / b)_{\text {hufs }}$ in Eq. (34), the Taylor series expansion of the exit plane wavefront for $\Theta_{0}$ in the vicinity of the transverse cusp point $(\mathrm{cp})$ is $[\underline{4}, \underline{5}]$

$$
\Phi(w, v)=-k w^{2} / 2 R a+k v^{2} / 2 S a-k g w v^{2} / 8 a^{2}+\ldots,
$$

where the dimensionless quantities $R$ and $g$ are taken to be positive and are functions of the central angle $\theta_{i}{ }^{0}$. When $\Delta_{V}=0$, the horizontal radius of curvature of the larger impact parameter rainbow supernumerary ray with $\varepsilon>0$ has the same value as for the sphere of Eq. (9). The wavefront of Eq. (27) differs from that of the Airy caustic of Eq. (8) by the addition of the third-order term proportional to $w v^{2}$ and the absence of the third-order term proportional to $w^{3}$. The absence of the term 
proportional to $w^{3}$ indicates that only one of the rainbow supernumerary rays participates in the caustic. The term proportional to $w v^{2}$ insures that the two skew rays participate, and smoothly joins the two local regions of identical curvature centered on each of these rays in the exit plane. For the existence of the hyperbolic umbilic caustic described in Section 5 , the coefficients of the $w^{3}$ and $w v^{2}$ terms in the outgoing wavefront in the exit plane must have the same sign. If they had opposite signs, the resulting caustic would instead be a three-cusped elliptic umbilic $[15,39]$. Since we already know that the coefficient of the $w^{3}$ term is negative in the vicinity of the rainbow, the coefficient of the $w v^{2}$ term must be negative, as well, which we denote here as $-g$.

Using the generalized ray tracing method described in $[7,8]$, the vertical radius of curvature of the supernumerary ray in the exit plane for $p=2$ spheroid scattering may be calculated and simplified to

$$
\begin{aligned}
S= & \left\{\cos \left(\theta_{t}^{0}\right) /[n \beta(1-\beta)]\right\}\{2 \beta \\
& \left.-\left[\left(1-A_{0}\right) /\left(2-\beta-A_{0}\right)\right]\right\}-\cos \left(\theta_{i}^{0}\right),
\end{aligned}
$$

where

$$
\beta=2(a / b)^{2}\left(1-A_{0}\right) \cos ^{2}\left(\theta_{t}{ }^{0}\right) .
$$

Again, using generalized ray tracing, the formula for $g$ is in general quite complicated. But when $\Theta_{0}=\Theta_{c p}$, most of the terms vanish and the expression reduces to

$$
g_{\mathrm{cp}}=8(a / b)^{2} \sin \left(\theta_{i}^{\mathrm{cp}}\right)\left(1-A_{\mathrm{cp}}\right)^{2} / \cos \left(\theta_{i}{ }^{\mathrm{cp}}\right) .
$$

As a sphere is distorted into an oblate spheroid, the sphere's axial caustics evolve into more complicated structures. An example of such an evolution is the glory caustic for scattering by a spherical bubble in water, which opens to become a four-cusped asteroid caustic for scattering by a spheroidal bubble [43-45]. The local transverse cusp caustic described in this section is a portion of the global asteroid caustic for spheroid glory scattering [2]. Only the two horizontally pointing cusps are visible, however, because the rays leading to what would have been the vertically pointing cusps are extinguished by total internal reflection at the $p=2$ interface [9]. In like manner, the virtual axial spike caustic of Eq. (20) for $p=2$ scattering by a sphere also becomes more complicated as the sphere evolves into a spheroid. The vertical radius of curvature $S$ of Eqs. (28) and (29) is again the distance from an effective point source on this more complicated caustic to the exit plane. As the spheroid radius ratio $a / b$ increases, $S$ becomes increasingly positive; it diverges and changes sign when $\beta=1$, signaling the shifting of the effective point source from the virtual portion to the real portion of this caustic, and then becomes decreasingly negative. The divergence of $S$ at $\beta=1$ produces a locally flat wavefront in the vertical direction, which causes the increased focusing on the horizontal axis near the cusp point seen in Fig. 1 of [37]. The behavior of the horizontal and vertical radii of curvature of the outgoing wavefront at the origin of the exit plane is shown in Fig. 2 as a function of $a / b$ and $\theta_{i}{ }^{0}$.

The divergence of $S$ when $\beta=1$ in Eq. (29) can be used to determine $\theta_{i}^{\text {cp }}$ if one knows $a / b$. One obtains $[\underline{7}, \underline{8}]$

$$
\begin{aligned}
\cos ^{2}\left(\theta_{i}^{\mathrm{cp}}\right)= & {\left[n^{2}-2(a / b)^{2}\left(n^{2}-1\right)\right]^{2} /\left\{4(a / b)^{2}\right.} \\
& \left.\times\left[n^{2}-(a / b)^{2}\left(n^{2}-1\right)\right]\right\} .
\end{aligned}
$$

The cusp point deflection angle $\Theta_{\text {cp }}$ is then obtained by substituting Eq. (31) into Eqs. (1) and (2). Alternatively, Eq. (29) can be used to determine $a / b$ if the cusp point deflection angle is known from experiment [1] and the central angle $\theta_{i}{ }^{\mathrm{cp}}$ is numerically obtained from it, giving [3]

$$
(a / b)_{\mathrm{cp}}=n /\left\{2 \cos \left(\theta_{t}^{\mathrm{cp}}\right)\left[n \cos \left(\theta_{t}^{\mathrm{cp}}\right)-\cos \left(\theta_{i}^{\mathrm{cp}}\right)\right]\right\} .
$$

Oblate spheroids whose $a / b$ ratio is in the range of $[\underline{30}, \underline{31}]$

$$
n /\left[2\left(n^{2}-1\right)\right]^{1 / 2} \leq a / b \leq\{n /[2(n-1)]\}^{1 / 2},
$$

corresponding to $\theta_{i}{ }^{\mathrm{cp}}=\pi / 2$ and $\theta_{i}{ }^{\mathrm{cp}}=0$, produce a transverse cusp caustic for $p=2$ scattering centered on the horizontal axis of the viewing screen to either side of the backscattering direction. When $a / b=$ $n /\left[2\left(n^{2}-1\right)\right]^{1 / 2}$ and $\theta_{i}{ }^{\mathrm{cp}}=\pi / 2$, the cusp points first form from the merging of the grazing incidence skew rays with the larger impact parameter supernumerary ray with grazing incidence. When $a / b=$ $\{n /[2(n-1)]\}^{1 / 2}$ and $\theta_{i}{ }^{\mathrm{cp}}=0$, the cusp points form

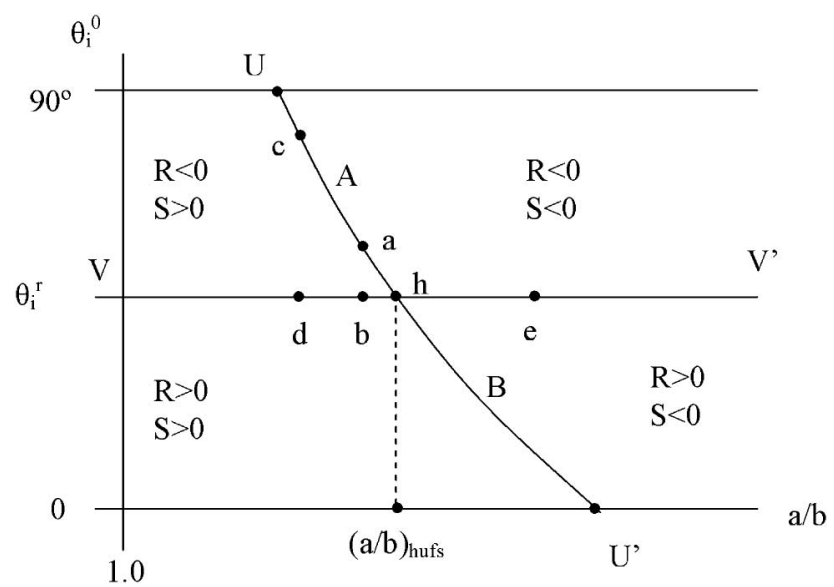

Fig. 2. The sign of the horizontal and vertical radii of curvature, $R$ and $S$, respectively, of the wavefront in the exit plane at the position of the central ray as a function of $\theta_{i}{ }^{\circ}$ and $a / b$. The horizontal radius $R$ of Eq. (15) is infinite on the line $V V^{\prime}$, and the vertical radius $S$ of Eq. (28) is infinite on the line $U U^{\prime}$. The point $h$ of Eq. (34), at which both radii of curvature diverge, is the condition for the hufs. The region above the line $V V^{\prime}$ is called region $\mathrm{A}$, and the region below $V V^{\prime}$ is called region $\mathrm{B}$. 
in the backscattered direction from the merging of the paraxial skew rays with the paraxial smaller impact parameter supernumerary ray. At this same value of $a / b$, the $p=2$ rainbow in the vertical direction occurs in the backscattering direction, as well, and the central portion of the global caustic formed by the joining of the cusps with the vertical rainbow shrinks to a single point, and is flanked by the Airy caustics still crossing the horizontal axis at the same locations as before (see Fig. $2 \mathrm{~d}$ of [9]). This gives the central portion a long, thin appearance that resembles a pair of lips, and which is known as a lips event in the terminology of catastrophe optics [15]. When $\theta_{i}{ }^{\mathrm{cp}}=\theta_{i}{ }^{r}$ for $p=2$ scattering, the spheroid with the radius ratio [2]

$$
(a / b)_{\mathrm{hufs}}=(n / 2)\left[3 /\left(n^{2}-1\right)\right]^{1 / 2}
$$

has its cusp point touching the increasingly curved rainbow caustic so as to form a V shape (see Fig. 2c of [9]). The coalescence of these four rays is the focal section of the hyperbolic umbilic caustic described in Section 5 .

Assuming $\Theta_{0}$ is in the vicinity of $\Theta_{\text {cp }}$ and the participating supernumerary ray is the larger impact parameter one, with a negative horizontal radius of curvature as described above (i.e., region $\mathrm{A}$ in Fig. 2), the scattered field can be reduced to Pearcey form. Equation (27) is substituted into Eq. (7), the $w$ integral is evaluated exactly, and the $v$ variable is scaled, giving $[\underline{4}, \underline{5}]$

$$
\begin{aligned}
E\left(\Delta_{H}, \Delta_{V}\right) \approx & -\exp \left[i\left(k a R \Delta_{H}^{2} / 2+\pi / 4\right)\right]\left\{(2 k a R)^{5 / 4}\right. \\
& \left./\left[(k Z)\left(\pi R^{2} g\right)^{1 / 2}\right]\right\} P(X, Y),
\end{aligned}
$$

with

$$
\begin{aligned}
& X=-(2 k a R)^{1 / 2}\left[\Delta_{H}-4 /(S R g)\right], \\
& Y=-2\left[(2 k a R)^{3 / 4} /\left(R^{2} g\right)^{1 / 2}\right] \Delta_{V} .
\end{aligned}
$$

In Eq. (35) and hereafter, we suppress the central phase $\Phi(0)$, the Fresenel coefficients, and the ray density amplification factors. If the participating supernumerary ray is the smaller impact parameter one, with a positive horizontal radius of curvature $R$ (i.e., region B in Fig. 2), the field of the cusp caustic is obtained by taking the negative of the complex conjugate of Eq. (35). No change in $X$ and $Y$ is needed for $R>0$ and $\Theta_{0}=\Theta_{\text {cp }}$, since the Pearcey function is symmetric in $Y$. The exponents of the spheroid size parameter $k a$, appearing in both the amplitude factor of Eq. (35) and the coordinates $X$ and $Y$ of Eqs. (36) and (37), agree with Table 2 of [16]. The shape of the caustic in the short wavelength limit for small $\Delta_{H}$ and $\Delta_{V}$ is obtained either by substituting Eqs. (36) and (37) into Eq. (25), or by setting both the gradient and the Hessian second derivative of Eq. (27) equal to zero. This second approach may be physically motivated as follows. The locus of zero Gaussian curvature of the phase function in the exit plane as a function of $w$ and $v$ is given by the vanishing of the Hessian second derivative. As is illustrated in Fig. 2 of [15] and Fig. 4.2 of [16], this locus is then mapped into the shape of the caustic on the viewing screen as a function of $\Delta_{H}$ and $\Delta_{V}$ by the vanishing of the gradient of the phase function. Using either method of calculation, the shape of the transverse cusp caustic is then

$$
\left(2 R^{2} g / 27\right)\left[\Delta_{H}-4 /(S R g)\right]^{3}=\Delta_{V}{ }^{2},
$$

independent of the size of the spheroid. The cusp opens toward the backscattering direction, in agreement with the experimental observations of [1]. If $\Theta_{0}=\Theta_{\text {cp }}$ so that the vertical radius of curvature $S$ of the outgoing wavefront of Eq. (28) at the origin of the exit plane diverges, the cusp point is located at the origin of the viewing screen, and $R$ and $g$ are evaluated using Eqs. (9) and (30), with the central ray $\theta_{i}$, , giving the opening rate

$$
\begin{aligned}
2 R^{2} g / 27= & (4 / 27)(a / b)^{2} \sin \left(\theta_{i}{ }^{\mathrm{cp}}\right) \cos \left(\theta_{i}{ }^{\mathrm{cp}}\right)[(1 \\
& \left.\left.-A_{\mathrm{cp}}\right) /\left(1-2 A_{\mathrm{cp}}\right)\right]^{2} .
\end{aligned}
$$

For scattering by a sphere in the physical optics model, both at the rainbow angle and at any generic angle other than the rainbow angle, the scattered intensity turns out to be independent of the location of the sphere's entrance and exit planes due to cancellations that occur in the factors $\left|R Q_{H}\right|$ and $\left|S Q_{V}\right|$ appearing in the scattered fields. Thus, choosing the entrance and exit planes to be tangent to the sphere, as in [25-27], is convenient in these situations. But for the transverse cusp, when the central ray is chosen so that $\Theta_{0}=\Theta_{\text {cp }}$ where $S$ diverges, isolated factors of the horizontal radius of curvature $R$ now appear in Eqs. (36) and (37) whose values depends on the location of the spheroid exit plane. If the exit plane were chosen to be tangent to the spheroid at $Z=a$, there would always occur one central scattering angle in the rainbow supernumerary region for which $R=0$. This is because, at this scattering angle, the $w$ axis of the exit plane perpendicularly intersects the near-zone real rainbow caustic of Eq. (19), corresponding to the effective point source of the outgoing rays lying in the exit plane. According to Eqs. (36) and (37), if this were to happen, the angular scale of the transverse cusp caustic would rapidly vary near this scattering angle. This is not observed experimentally. It is not evident to us that these isolated factors of $R$ within the Pearcey function would be canceled by the multiplicative Fresnel coefficients and ray density amplification factors we have chosen to neglect here. Thus, moving the spheroid exit plane back to $Z=0$, as was done in [28,29], insures that it will never perpendicularly intersect the near-zone real or virtual rainbow caustic and, thus, prevents 
the $R=0$ condition from occurring. This choice of exit plane was used in deriving Eqs. (9)-(13), (28), and (30).

\section{Hyperbolic Umbilic Caustic}

The hyperbolic umbilic caustic is a four-ray-to-tworay-to-zero-ray transition whose canonical diffraction integral is [15]

$$
E(X, Y, Z)=\int_{-\infty}^{\infty} \mathrm{d} s \int_{-\infty}^{\infty} \mathrm{d} t \exp \left[i\left(s^{3}+t^{3}-Z s t-X s-Z t\right)\right] .
$$

For $p=2$ scattering of a plane wave by an oblate spheroid with $\Theta_{0}$ in the vicinity of this caustic in region A of Fig. 2, the Taylor series expansion of the exit plane outgoing wavefront for small values of $w$ and $v$ contains the third-order terms of both the Airy and transverse cusp caustics [6]:

$$
\begin{aligned}
\Phi(w, v)= & -k w^{2} / 2 R a-k h w^{3} / 3 a^{2}+k v^{2} / 2 S a \\
& -k g w v^{2} / 8 a^{2}+\ldots
\end{aligned}
$$

The dimensionless quantities $R, h$, and $g$ are positive and are functions of the central angle $\theta_{i}{ }^{0}$. The presence of the terms proportional to $w^{3}$ and $w v^{2}$ signals the participation in the caustic of both rainbow supernumerary rays and both skew rays. The vertical radius of curvature $S$ is also a function of $\theta_{i}{ }^{0}$. Again, for constant $\theta_{i}{ }^{0}, S$ is positive when $a / b<$ $(a / b)_{\mathrm{cp}}$, it diverges and changes sign when $a / b=$ $(a / b)_{\mathrm{cp}}$, and it is negative when $a / b>(a / b)_{\mathrm{cp}}$.

The shape of the hyperbolic umbilic caustic in the short wavelength limit can be obtained by setting both the gradient and the Hessian second derivative of Eq. (41) equal to zero. Taylor series expanding the result about the origin of the viewing screen in powers of $\Delta_{H}$ and $\Delta_{V}$ in region A of Fig. $\underline{2}$, when neither $R$ nor $S$ diverges, gives

$$
\Delta_{H}=-\left[1 /\left(4 R^{2} h\right)\right]+\left(8 h^{2} S^{2} / g\right) \Delta_{V}^{2} /(S / R+8 h / g)^{2}
$$

for the parabolically curved Airy portion of the hyperbolic umbilic caustic and

$$
\begin{gathered}
\left(2 S^{2} g / 27\right)\left[\Delta_{H}-\left(4 / S^{2} g\right)(S / R+4 h / g)\right]^{3} \\
/(S / R+8 h / g)^{2}=\Delta_{V}{ }^{2}
\end{gathered}
$$

for the transverse cusp portion, independent of the size of the spheroid. For region B in Fig. 2, the horizontal radius of curvature is positive and, thus, the quantity $R$ in Eqs. (42) and (43) should be replaced by $-R$.

Because catastrophe optics methods give quantitative information only in the immediate neighborhood of $\Theta_{o}$, Eqs. (42) and (43) quantitatively describe both branches of the hyperbolic umbilic caustic if the deflection angle of the cusp point and the Airy caustic are angularly close to each other, as is the case for points $\mathrm{a}$ and $\mathrm{b}$ in Fig. 2 . If the central angle is chosen so that $\Theta_{0}=\Theta_{\text {cp }}$, the quantities $R, g$, and $h$ are evaluated at $\theta_{i}^{\text {cp }}$, the angles $\Delta_{H}$ and $\Delta_{V}$ are taken to be with respect to $\Theta_{\mathrm{cp}}$, the transverse cusp caustic has its point at $\Delta_{H}=0, \Delta_{V}=0$, and is identical to the $S \rightarrow \infty$ limit of Eq. (38). The Airy caustic crosses the horizontal axis at $\Delta_{H}=-1 / 4 R^{2} h$. On the other hand, if the central angle is chosen so that $\Theta_{0}=\Theta_{r}$, the quantities $S, g$, and $h$ are evaluated at $\theta_{i}{ }^{r}$, the angles $\Delta_{H}$ and $\Delta_{V}$ are taken to be with respect to $\Theta_{r}$, and the parabolically curved Airy caustic crosses the horizontal axis at $\Delta_{H}=0$. The cusp point occurs at $\Delta_{H}=4 /\left[\left(S^{2} g\right)(S / R+4 h / g)\right]$. But when the deflection angle of the cusp point and Airy caustic have a larger angular separation, as is the case for points $\mathrm{c}$ and $\mathrm{d}$ in Fig. 2, only one of the branches of the hyperbolic umbilic caustic is described quantitatively by Eqs. (42) and (43). The choice $\Theta_{0}=\Theta_{c p}$ provides a quantitative prediction of the cusp shape, but gives only an approximation to the Airy caustic's shape, and the choice $\Theta_{0}=\Theta_{r}$ provides a quantitative prediction of the Airy caustic's shape, but gives only an approximation to the cusp's shape.

When $a / b=(a / b)_{\text {hufs }}$ and the central ray is chosen to be the rainbow's Descartes ray (point h in Fig. 2 ), both $R$ and $S$ simultaneously diverge. One obtains the V-shaped hyperbolic umbilic focal section for small $\Delta_{H}$ and $\Delta_{V}$ :

$$
\pm(8 h / g)^{1 / 2} \Delta_{V}=\Delta_{H},
$$

independent of the size of the spheroid, where $\Delta_{H}$ is nonnegative, $g$ and $h$ are evaluated at $\theta_{i}{ }^{r}$, and the angles $\Delta_{H}$ and $\Delta_{V}$ are taken to be with respect to $\Theta_{r}$.

For each of the hyperbolic umbilic caustics observed for side-on scattering by an oblate spheroid, the locus of zero Gaussian curvature of the phase function in the exit plane as a function of $w$ and $v$ is the two disjoint branches of a hyperbola, as shown in Fig. 3 , which we denote as branch 1 and branch 2 . For the transverse cusp of Section 4, the locus is a parabola, and for the elliptic umbilic caustic it is an ellipse. When the vertical radius of curvature of the wavefront is positive, such as at point $d$ in Fig. 2 , the caustic image on the viewing screen of branch 1 of the hyperbola is the cusp caustic of Eq. (43), and the caustic image of branch 2 is the parabolic shaped fold caustic of Eq. (42). This is illustrated in Fig. 3e of [15] and Fig. 2.5e of [16]. In the experiments of [1], one can imagine keeping the viewing screen centered on the Descartes rainbow angle in the horizontal direction, and continuously increasing $a / b$ from point $d$ in Fig. 2 through the hufs of point h, to point e, where the vertical radius of curvature is negative. At the hufs, each of the branches of the hyperbola in the exit plane evolves into a V-shape whose points now touch, 
forming an overall $\mathrm{X}$ shape. For larger $a / b$, the zero Gaussian curvature locus in the exit plane relaxes back to the original hyperbola, with its two disjoint branches. The caustic image on the viewing screen of this progression is the parabola and cusp merging into two completely overlapping copies of the Vshaped hufs of Eq. (44), and then relaxing back into the parabolic fold and cusp caustics of Eqs. (42) and (43), except now there has been an interchange of identities. At point e in Fig. 2, the parabolic fold on the viewing screen is the caustic image of branch 1 of the hyperbola in the exit plane, and the cusp is the image of branch 2. This exchange of identities of the cusp and fold is suggested by the crossing of the two sheets of the caustic in Fig. 3e of [15] and Fig. 2.5e of [16], and will later prove important in understanding the scattering caustic's global behavior as a function of $a / b$.

For region A of Fig. 2, where the horizontal radius of curvature of the central ray in the exit plane is negative, the field of the hyperbolic umbilic caustic is obtained by substituting Eq. (41) into Eq. (7). The result can then be reduced to the canonical form of Eq. (40) by the substitution

$$
\begin{aligned}
X= & (k a / 2)^{2 / 3}(3 / h)^{1 / 3}\left\{\left[\Delta_{H}-(8 h / g)^{1 / 2} \Delta_{V}\right]\right. \\
& \left.+\left(3 / 16 S^{2} h\right)(S / R-8 h / g)(S / R+8 h / 3 g)\right\},
\end{aligned}
$$

$$
\begin{aligned}
Y= & (k a / 2)^{2 / 3}(3 / h)^{1 / 3}\left\{\left[\Delta_{H}+(8 h / g)^{1 / 2} \Delta_{V}\right]\right. \\
& \left.+\left(3 / 16 S^{2} h\right)(S / R-8 h / g)(S / R+8 h / 3 g)\right\},
\end{aligned}
$$

$$
Z=(k a / 2)^{1 / 3}(3 / h)^{2 / 3}(1 / 2 S)(S / R+8 h / g) .
$$

The exponents of $k a$ appearing in Eqs. (45)-(47) agree with Table 2 of [16]. For region B in Fig. 2 , where the horizontal radius of curvature of the central ray in the exit plane is positive, the quantity $R$ in Eqs. (45)-(47) should be replaced by $-R$.

The diffraction integral of Eqs. (7) and (41) for region A of Fig. 2 can be evaluated approximately for $\Delta_{V}=0$ by using the method of stationary phase. We first choose the central scattering angle as $\Theta_{0}=\Theta_{r}$. We also assume that both $\Delta_{H}$ and $\Delta_{\mathrm{cp}}$ are small, where

$$
\Delta_{\mathrm{cp}}=16 h /(g S)^{2},
$$

so that $\Theta_{c p}$ is in the neighborhood of $\Theta_{r}$, as well. Then, if $\Delta_{H}$ is in the interval $\Delta_{H}<0$, the viewing screen point is in the zero-ray region and, if $0<\Delta_{H}<\Delta_{\mathrm{cp}}$, the viewing screen point is in the two-ray region, where the only participating rays are the rainbow supernumerary rays. On the other hand, if $\Delta_{\text {cp }}<\Delta_{H}$, the viewing screen point is in the four-ray region, where the two skew rays parti-

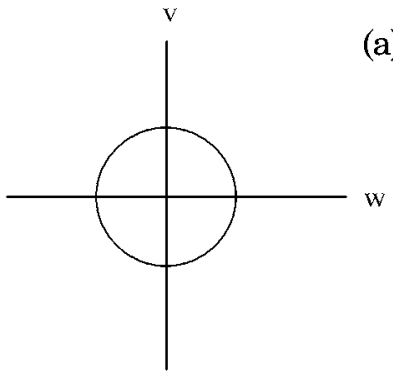

(a)
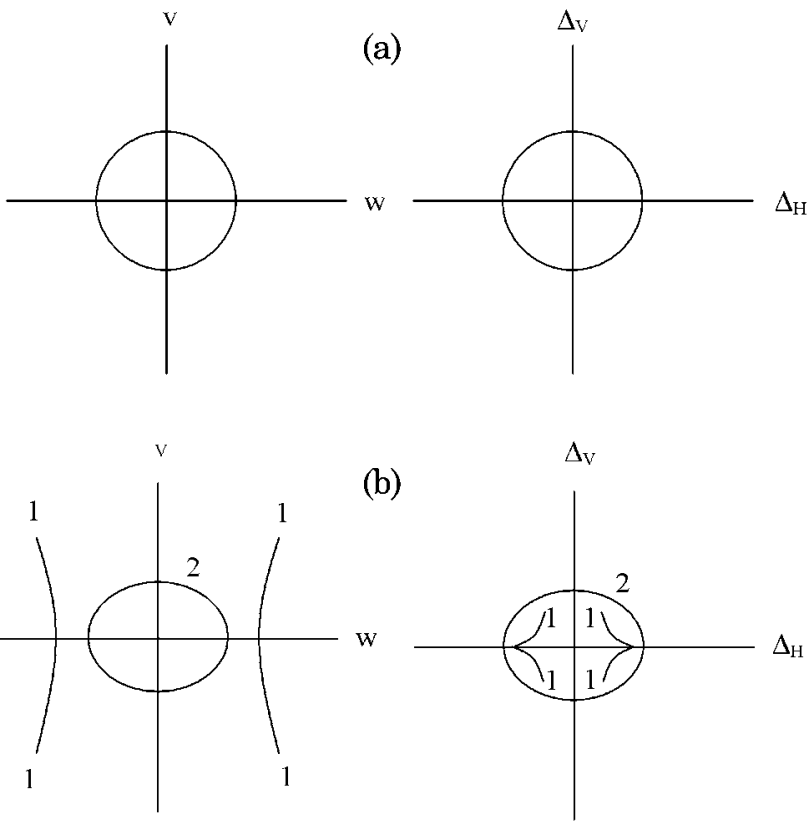

(b)
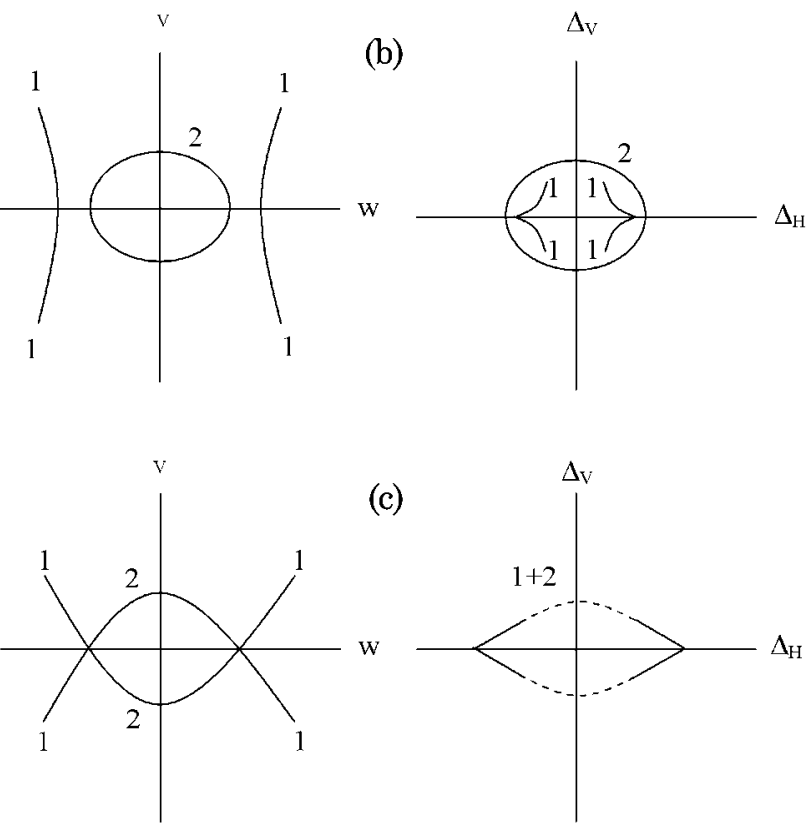

(c)
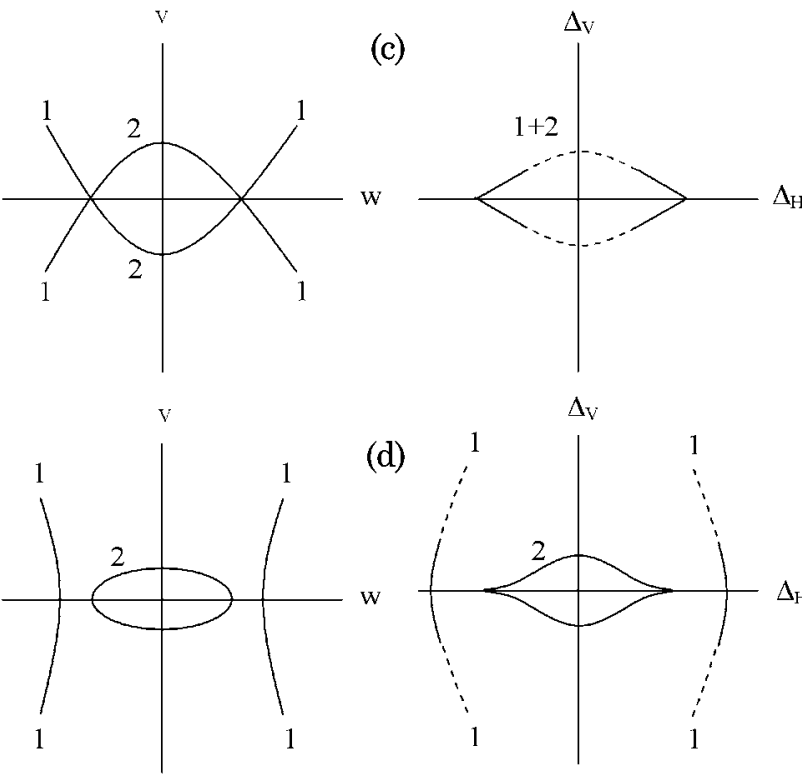

(d)

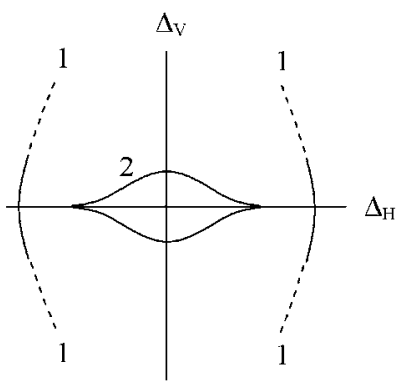

Fig. 3. Locus of zero Gaussian curvature as a function of $w$ and $v$ in the spheroid exit plane (left-hand figures) and its caustic image as a function of $\Delta_{H}$ and $\Delta_{V}$ on the far-zone viewing screen (righthand figures). The radius ratio for (a) is $a / b=1$, and $a / b$ progressively increases in (b), (c), and (d), where (c) corresponds to the hufs condition of Eq. (34). The branches of the zero Gaussian curvature locus and their respective caustic images are labeled 1 and 2. At the hufs condition, the part of the zero Gaussian curvature locus that is mapped to the vertical portion of the rainbow detaches from the part of the locus that is mapped into the horizontal portion of the rainbow and attaches to the part of the locus that is mapped into the transverse cusp caustics. The dashed lines on the caustics in (c) and (d) denote regions of greatly reduced intensity in Figs. 4 and $\underline{5}$.

cipate, as well. When $\Delta_{H}$ is not too close to either zero or $\Delta_{\mathrm{cp}}$, the scattered field in the stationary phase approximation is given by either the two or four individual ray contributions added together: 


$$
\begin{aligned}
& E\left(\Delta_{H}, 0\right) \approx[(k a / k Z)](2 / g)^{1 / 2}\left(\Delta_{H} \Delta_{\mathrm{cp}}\right)^{-1 / 4} \\
& \quad \times\left[1+\left(\Delta_{H} / \Delta_{\mathrm{cp}}\right)^{1 / 2}\right]^{-1 / 2} \exp (-2 i k a \\
& \left.\quad \times \Delta_{H}{ }^{3 / 2} / 3 h^{1 / 2}\right)-[i(k a / k Z)](2 / g)^{1 / 2} \\
& \quad \times\left(\Delta_{H} \Delta_{\mathrm{cp}}\right)^{-1 / 4}\left[1-\left(\Delta_{H} / \Delta_{\mathrm{cp}}\right)^{1 / 2}\right]^{-1 / 2} \\
& \quad \times \exp \left(2 i k a \Delta_{H}^{3 / 2} / 3 h^{1 / 2}\right) \\
& \quad \text { for } 0<\Delta_{H}<\Delta_{\mathrm{cp}}
\end{aligned}
$$

and

$$
\begin{aligned}
E\left(\Delta_{H}, 0\right) \approx & {[(k a / k Z)]\left(2 / g \Delta_{H}\right)^{1 / 2}[1} \\
& \left.+\left(\Delta_{\mathrm{cp}} / \Delta_{H}\right)^{1 / 2}\right]^{-1 / 2} \exp (-2 i k a \\
& \left.\times \Delta_{H}{ }^{3 / 2} / 3 h^{1 / 2}\right)-[(k a / k Z)]\left(2 / g \Delta_{H}\right)^{1 / 2} \\
& \times\left[( 1 - ( \Delta _ { \mathrm { cp } } / \Delta _ { H } ) ^ { 1 / 2 } ] ^ { - 1 / 2 } \operatorname { e x p } \left(2 i k a \Delta_{H} 3 / 2\right.\right. \\
& \left./ 3 h^{1 / 2}\right)-[2 i(k a / k Z)]\left(2 / g \Delta_{H}\right)^{1 / 2} \\
& \times\left(1-\Delta_{\mathrm{cp}} / \Delta_{H}\right)^{-1 / 2} \exp i k a\left(\Delta_{\mathrm{cp}} / h\right)^{1 / 2} \\
& \times\left(\Delta_{H}-\Delta_{\mathrm{cp}} / 3\right) \\
& \text { for } \Delta_{\mathrm{cp}}<\Delta_{H} .
\end{aligned}
$$

In Eqs. (49) and (50), the first two terms correspond to the smaller and larger impact parameter supernumerary rays, respectively, and in Eq. (50), the third term is the field of the two skew rays. All the terms in Eqs. (49) and (50) are proportional to $k a$ because they are contributions of individual geometrical rays. When $\Delta_{H}$ is much closer to zero than it is to $\Delta_{\mathrm{cp}}$, by using Eq. (23), the scattered field for $\Delta_{V}=0$ in either the two-ray or zero-ray region reduces to

$$
\begin{aligned}
E\left(\Delta_{H}, 0\right) \approx & \left\{(2)^{3 / 2} \pi^{1 / 2} /\left[(k Z) g^{1 / 2} h^{1 / 12} \Delta_{\mathrm{cp}}{ }^{1 / 4}\right]\right\} \\
& \times \exp (-i \pi / 4)\left\{( k a ) ^ { 7 / 6 } A i \left[-(k a)^{2 / 3}\right.\right. \\
& \left.\times \Delta_{H} / h^{1 / 3}\right]-i\left[h^{1 / 6}(k a)^{5 / 6} /\left(2 \Delta_{\mathrm{cp}}{ }^{1 / 2}\right)\right] \\
& \left.\times A i^{\prime}\left[-(k a)^{2 / 3} \Delta_{H} / h^{1 / 3}\right]\right\}
\end{aligned}
$$

For scattering by a sphere, the $A i^{\prime}$ dependence of the scattered fields is produced by the variation of the Fresnel coefficients and the wavefront amplitude across the exit plane $[42,46]$. For spheroid scattering, this variation has not been taken into account, and the $A i^{\prime}$ dependence in Eq. (51) is produced by the distortion of the supernumerary ray amplitudes in Eq. (49) due to the presence of the nearby cusp point. On the other hand, when $\Delta_{H}$ is much closer to $\Delta_{c p}$ than it is to zero, by using Eq. (26), the scattered field in either the two-ray or four-ray region reduces to

$$
\begin{aligned}
& E\left(\Delta_{H}, 0\right) \approx\left\{(k a) /\left[(k Z)\left(g \Delta_{\mathrm{cp}}\right)^{1 / 2}\right]\right\} \exp [-2 i(k a) \\
& \left.\times \Delta_{H}^{3 / 2} / h^{1 / 2}\right]-\left\{2(k a)^{5 / 4} /\left[(k Z) \pi^{1 / 2} g^{1 / 2}\right.\right. \\
& \left.\left.\times\left(\Delta_{\mathrm{cp}} h\right)^{1 / 8}\right]\right\} \exp (i \pi / 4) \\
& \times \exp \left[2 i(k a) \Delta_{H}^{3 / 2} / h^{1 / 2}\right] P(X, 0),
\end{aligned}
$$

where

$$
X=(k a)^{1 / 2}\left(\Delta_{\mathrm{cp}}-\Delta_{H}\right) /\left(\Delta_{\mathrm{cp}} h\right)^{1 / 4} .
$$

Because we have assumed that the horizontal radius of curvature is negative here, the first term in Eq. (52) is the lower supernumerary impact parameter ray whose field is proportional to $k a$. The second term proportional to $(k a)^{5 / 4}$, as in Eq. (31), is the confluence of the higher impact parameter supernumerary ray with the two skew rays to form the transverse cusp caustic. When the horizontal radius of curvature is positive, the fields are obtained by taking the negative of the complex conjugate of Eqs. (49)-(52). In this case, the smaller impact parameter supernumerary ray participates in the transverse cusp and the larger supernumerary ray is the spectator.

For the special case of the hyperbolic umbilic focal section, the diffraction integral can be evaluated exactly in terms of Airy functions when $\Theta_{0}=\Theta_{r}$ and $a / b=(a / b)_{\text {hufs }}$ by substituting Eq. (41) into Eq. (7), deleting the terms proportional to $w^{2}$ and $v^{2}$ because the horizontal and vertical radii of curvature diverge there, and by making a skew transformation to new variables $s$ and $t$, which separate the two-dimensional integral into two identical onedimensional integrals. The result for $p=2$ scattering is [] $]$

$$
\begin{gathered}
E\left(\Delta_{H}, \Delta_{V}\right) \approx i\left\{2^{7 / 2} \pi(k a / 2)^{4 / 3} /\left[(k Z) g^{1 / 2} h^{1 / 6}\right]\right\} \\
\quad \times A i\left\{-(k a / 2)^{2 / 3}\left[\Delta_{H}-(8 h / g)^{1 / 2} \Delta_{V}\right] / h^{1 / 3}\right\} \\
\quad \times A i\left\{-(k a / 2)^{2 / 3}\left[\Delta_{H}+(8 h / g)^{1 / 2} \Delta_{V}\right] / h^{1 / 3}\right\} .
\end{gathered}
$$

The argument of the Airy functions is zero on the Vshape lines of Eq. (44), and the field of each Airy function is oscillatory inside the V-shape, where $\Delta_{H}>0$, thus producing a complicated diffraction pattern (see Fig. 5 of [6]). The exponents of $k a$ appearing in both the amplitude of Eq. (54) and in the arguments of the Airy functions agree with Table 2 of [16]. For $p=2$ scattering at the hufs condition, both $R$ and $S$ of Eqs. (9) and (28) diverge, whereas both $h$ and $g$ of Eqs. $(\overline{10})$ and $(\overline{30})$ remain finite. Either way, the opening rate of the hufs is found to be [6]

$$
(8 h / g)_{\mathrm{hufs}}=\lim _{R, S \rightarrow \infty} S / R=12 / n^{2} .
$$

In [9], the evolution of the $p=2$ global caustic as a function of $a / b$ in the range given by Eq. (33) is qualitatively explained in terms of one of the unfoldings [47] of the higher caustic $X_{9}$. This unfolding explains why, for $a / b<(a / b)_{\text {hufs }}$, the portions of the rainbow in the vertical and horizontal directions are connected to each other, while the transverse cusps are separate; at $a / b=(a / b)_{\text {hufs }}$, the vertical portions of the rainbow detach from the horizontal portions; and, for $a / b>(a / b)_{\text {hufs, }}$, the vertical portions of the rainbow and the transverse cusps are now connected 
to each other, while the horizontal portions of the rainbow are separate. The unfolding of $X_{9}$ is pictorially illustrated in Figs. 2 and 8 of [9], Fig. 2 of [2], and Fig. A2.2 of [47]. It can be understood in the context of spheroid scattering as follows and is illustrated here as Fig. 3. In Fig. 3(a), for scattering by a spherical water droplet, the global caustic on a viewing screen placed in the backscattering direction is the circular-shaped rainbow, as in Fig. 2a of [9], which we can consider as the caustic image of a circle of zero Gaussian curvature on some suitably defined exit plane with horizontal axis $w$ and vertical axis $v$. There is no $p=2$ backscattered axial caustic because backscattering of geometric rays by a water droplet does not occur. As $a / b$ is increased into the range of Eq. (33), a pair of cusps appears on the viewing screen inside the vertically flattened rainbow, as in Fig. 2b of [9]. In Fig. 3(b) here, the zero Gaussian curvature locus on the exit plane for this situation has three disjoint segments. One of the segments is what originally was the circular locus for a sphere, but which has now been vertically flattened so as to become approximately elliptical. It crosses the $\pm w$ axis, looking locally like a pair of branch two portions of hyperbolas leading to the horizontal portions of the rainbow, and it crosses the $\pm v$ axis leading to the vertical portions of the rainbow. To either side of this vertically flattened approximate ellipse are the other two segments of the zero Gaussian curvature locus in the exit plane. These segments locally look like a pair of branch 1 portions of hyperbolas, matching the branch 2 portions of the first segment of zero curvature, and leading to the two transverse cusps. As was described above, when $a / b=(a / b)_{\text {hufs }}$ and illustrated in Fig. 3(c) here, each set of branches 1 and 2 in the exit plane touch in an X-shape and are joined together on the $\pm v$ axis by the continuation of branch 2 . The resulting viewing screen caustic is two completely overlapping V-shaped hufs sections joined by the vertical portions of the rainbow, as in Fig. 2c of [9]. As $a / b$ is further increased and shown in Fig. 3(d) here, the zero Gaussian curvature locus on the exit plane reverts to an increasingly flattened approximate ellipse, which we still denote as branch 2 , flanked by a pair of branch 1 portions of the hyperbolas. But the caustic images on the viewing screen of branches 1 and 2 in the exit plane have interchanged. The images of the two branch 1 portions are now the locally parabolic-shaped fold caustics of Fig. 2d of [9]. Between them is the caustic image of branch 2 , which still contains the vertical portion of the rainbow, but now also contains the two transverse cusp caustics. Since the vertical portion of the rainbow has detached from the horizontal portion of the rainbow and is now connected to the transverse cusps, this new structure shrinks as $a / b$ is further increased to the lips event described in Section 4. It is then extinguished and only the horizontal portions of the rainbow remain.

\section{Computed Caustic Patterns in the Scattered Intensity}

The $p=2$ portion of the scattered intensity was numerically computed using the method described in [18] for side-on incidence of a plane wave on an oblate spheroid. For the geometry illustrated in Fig. 1, the incident plane wave propagates in the positive $z$ direction with $\lambda=0.5145 \mu \mathrm{m}$, and is polarized either in the $y$ direction or is unpolarized. The oblate spheroid has refractive index $n=1.334+i\left(1.2 \times 10^{-9}\right)$, horizontal radius $a=6 \mu \mathrm{m}$, and various values of the vertical radius $b$. It should be noted that, in [18], the standard definition of spheroidal coordinates has the $z$ axis coincident with the particle's symmetry axis, rather than being the direction of the incoming light, which is perpendicular to the spheroid's symmetry axis for side-on incidence. As a result, the intensity was computed as a function of the spheroidal coordinate system scattering angles and then had to be converted to the scattering angles $\Theta$ and $\Phi$ for the orientation of the axes in Fig. 1. For vertically polarized incident light, the scattered intensity as a function of the scattering angles $\Theta$ and $\Phi$ on a distant viewing screen centered at $\Theta=180^{\circ}$ is shown as a polar plot in Figs. 4(a)-4(f) for $a / b=1.0001,1.05$, $1.15,1.25,1.30$, and 1.36 , respectively. In these figures, the $y$ axis of Fig. 1 is vertical $\left(\Phi=90^{\circ}, 270^{\circ}\right)$ and the $z x$ plane is horizontal $\left(\Phi=0^{\circ}, 180^{\circ}\right)$. Similarly, Figs. 5(a)-5(f) show the scattered intensity as a function of $\Theta$ and $\Phi$ for the same $a / b$ ratios for unpolarized incident light. In Fig. 5(a), for scattering of unpolarized light by a sphere, the main peak of the circular rainbow caustic appears prominently at $\Theta=$ $143.60^{\circ}$ in the horizontal direction and $\Theta=143.55^{\circ}$ in the vertical direction. For comparison, Eqs. (1) and (15) in the short wavelength limit give the Descartes angle for the $p=2$ rainbow with $n=1.334$ as $\Theta_{r}=138.07^{\circ}$. But, according to Eq. (21), the first rainbow peak for the size parameter used here is shifted in Airy theory to $\Theta=143.72^{\circ}$, in good agreement with the results of Fig. 5(a). The residual deviation is comparable to that found in [18]. In Fig. 4(a), for vertically polarized incident light, scattering in the horizontal direction is TE polarized and the rainbow is well approximated by an Airy function. But scattering in the vertical direction is TM polarized and the rainbow there is well approximated by the derivative of an Airy function. The TM intensity is noticeably dim because the transmitted angle into the sphere for the rainbow ray is near the Brewster angle, and produces only a very weak internal reflection. According to Airy theory [42], the main peak of the $A i^{\prime}$ function should occur at $\Theta=151.10^{\circ}$ for this size parameter and agrees well with the angle $\Theta=$ $150^{\circ}$ observed in Fig. 4(a). The transition of the rainbow intensity from the dominant Airy function behavior to the dominant derivative of an Airy function behavior at about $\Phi=45^{\circ}, 135^{\circ}$ is also clearly evident in Fig. 4(a). As a side note, the results of Fig. 4(a) suggest that, if the atmospheric $p=2$ rainbow is viewed just before sunset through a polarizing 


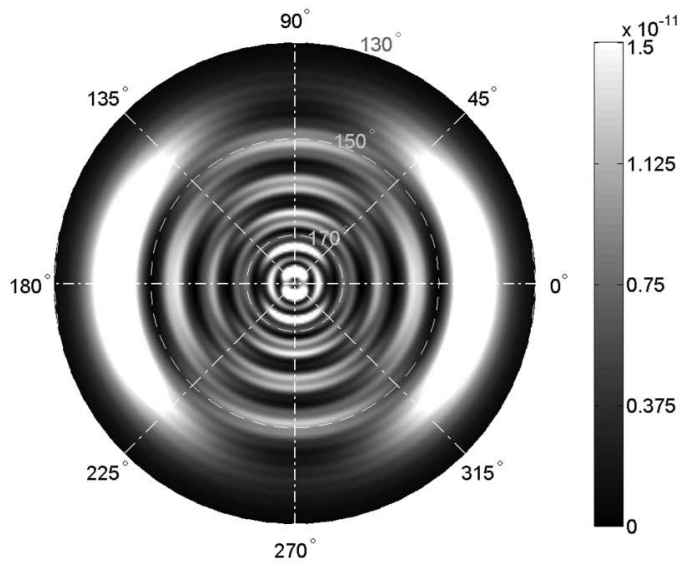

(a)

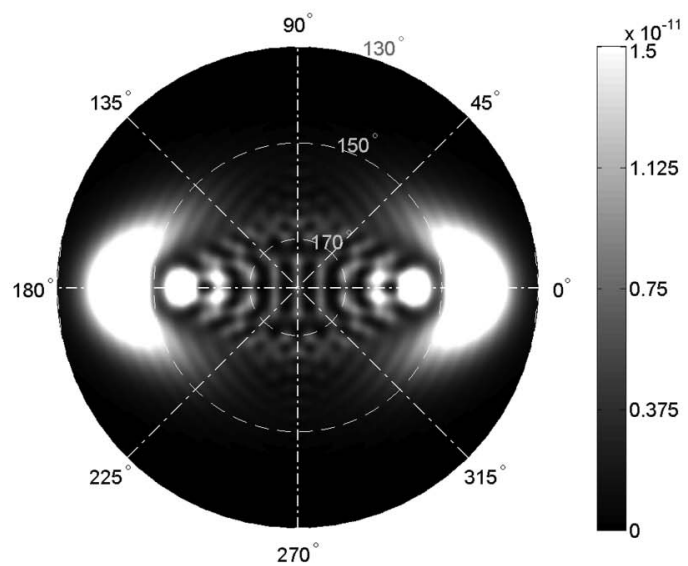

(c)

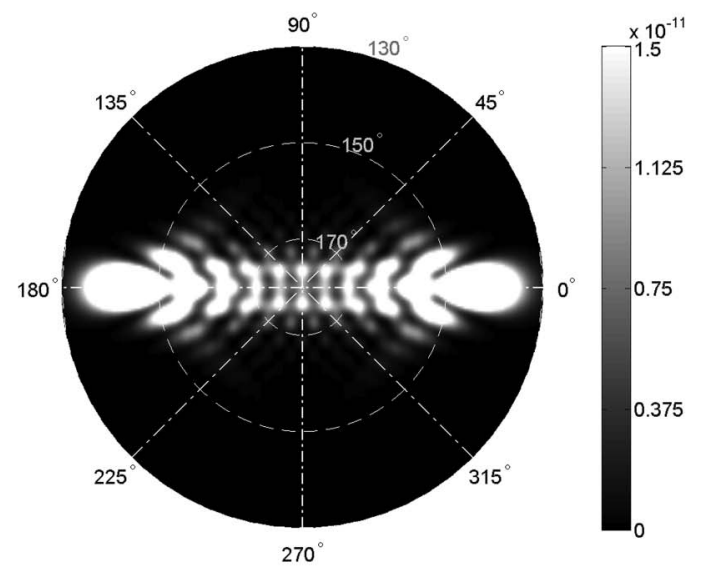

(e)

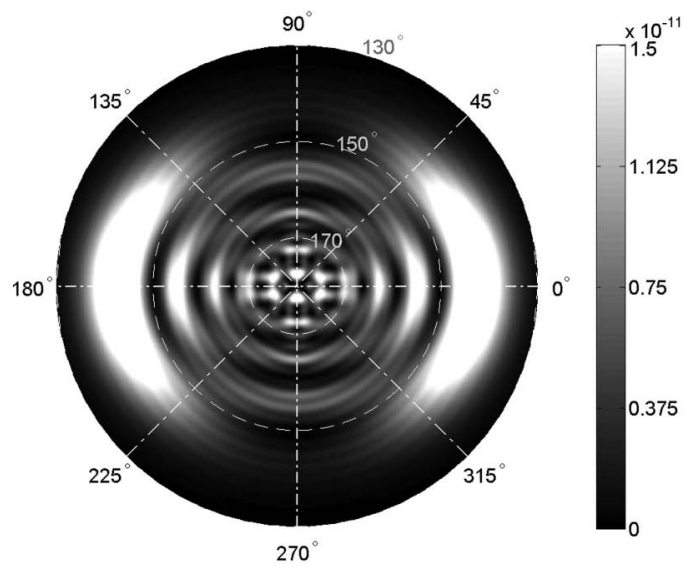

(b)

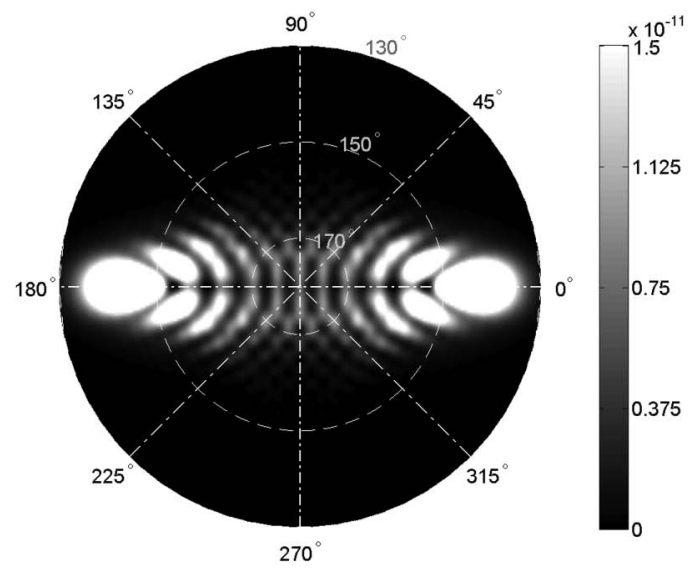

(d)

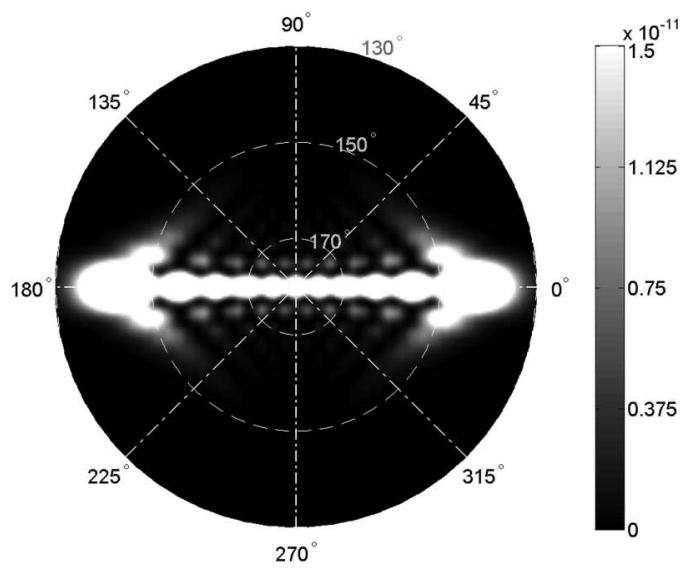

(f)

Fig. 4. Polar plot of the intensity as a function of the angles $\Theta$ and $\Phi$ for a vertically polarized incident plane wave with wavelength $\lambda=0.5145 \mu \mathrm{m}$ scattered by an oblate spheroid with refractive index $n=1.334+i\left(1.2 \times 10^{-9}\right)$, horizontal radius $a=6.0 \mu \mathrm{m}$, and horizontalto-vertical radius ratio (a) $a / b=1.0001$, (b) $a / b=1.05$, (c) $a / b=1.15$, (d) $a / b=1.25$, (e) $a / b=1.30$, and (f) $a / b=1.36$.

filter held by an observer, its intensity along the rainbow arc should depend on $\Phi$. Depending on the orientation of the polarizing filter, it should be brighter at the top of the arc and dimmer at the sides or dimmer at the top of the arc and brighter at the sides.

As a general rule of thumb, in the full Mie intensity for scattering by a sphere, there are about $k a$ inten- sity peaks in the angular interval $0^{\circ} \leq \Theta \leq 180^{\circ}$, giving an average angular size per peak of roughly $180^{\circ} / k a$. Some peaks, such as the main rainbow peak, are wider than this, while other peaks, such as the sequence of $p=0,1$ interference peaks in the forward hemisphere, may be a bit narrower. In Fig. 5(a), for unpolarized incident light, there are 


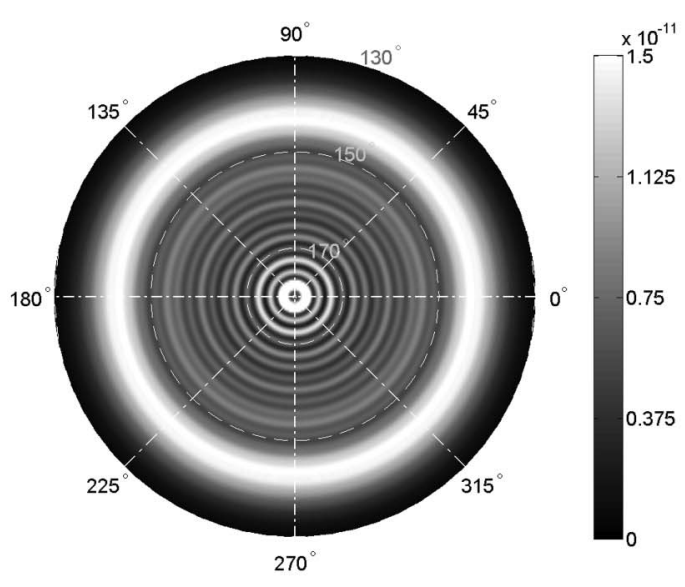

(a)

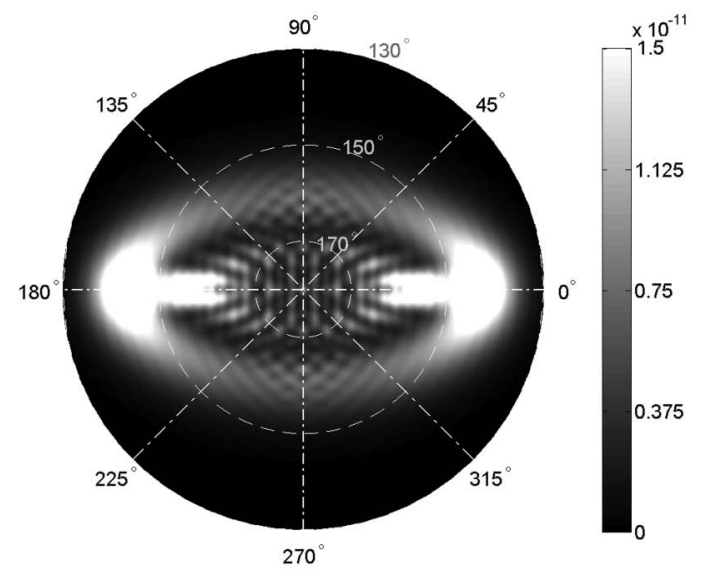

(c)

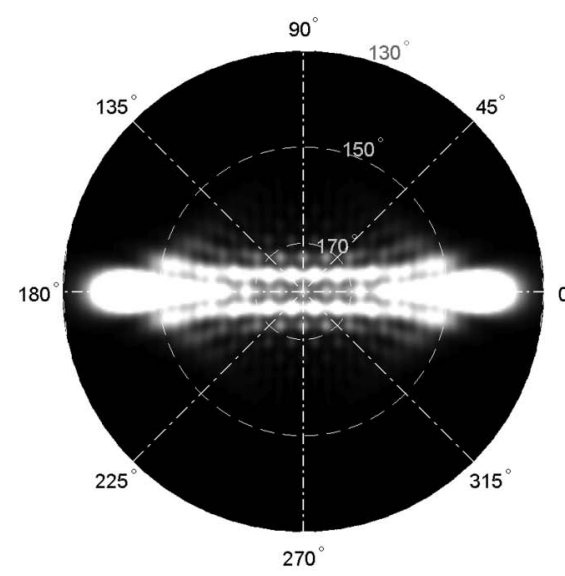

(e)

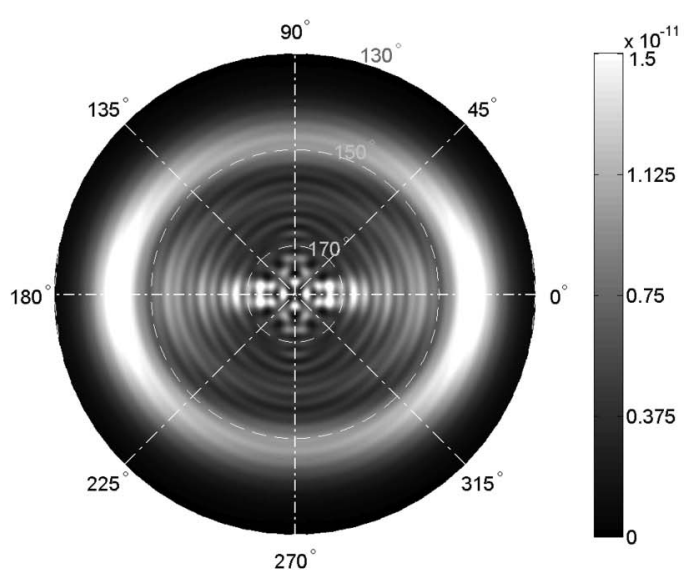

(b)

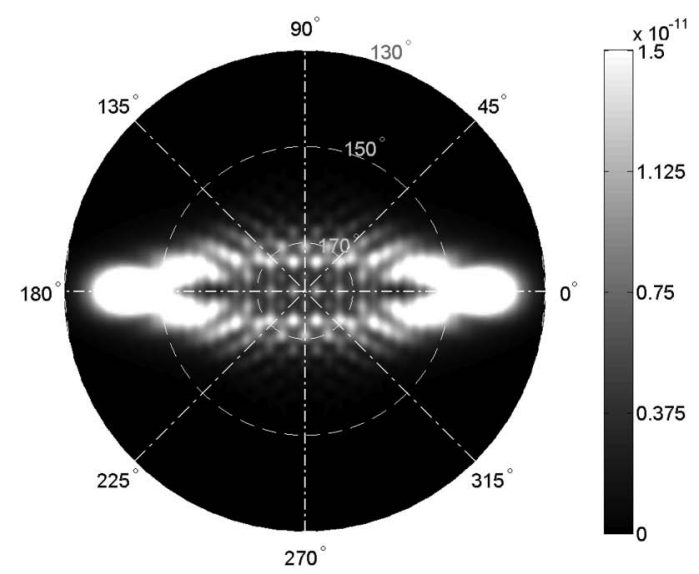

(d)

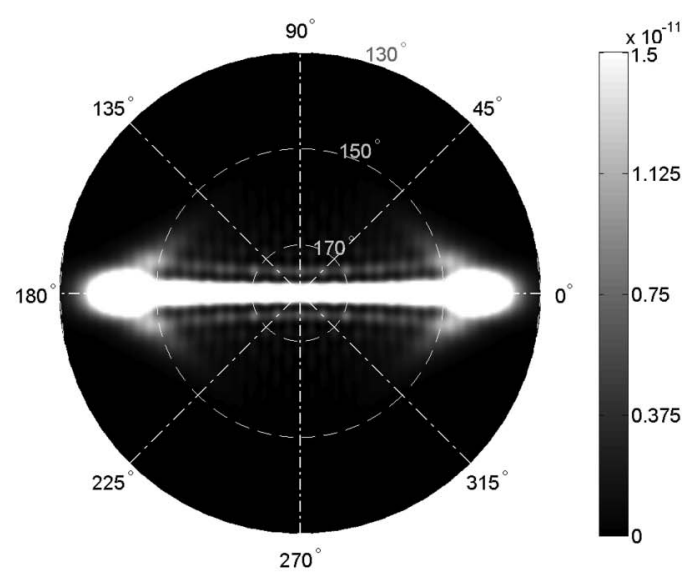

(f)

Fig. 5. Polar plot of the intensity as a function of the angles $\Theta$ and $\Phi$ for an unpolarized incident plane wave with wavelength $\lambda=$ $0.5145 \mu \mathrm{m}$ scattered by an oblate spheroid with refractive index $n=1.334+i\left(1.2 \times 10^{-9}\right)$, horizontal radius $a=6.0 \mu \mathrm{m}$, and horizontal-to-vertical radius ratio (a) $a / b=1.0001$, (b) $a / b=1.05$, (c) $a / b=1.15$, (d) $a / b=1.25$, (e) $a / b=1.30$, and (f) $a / b=1.36$.

13 peaks in the interval $143.60^{\circ} \leq \Theta \leq 180.0^{\circ}$, which compares well to the rule of thumb prediction of 15 peaks. However, in Fig. 4(a), for vertically polarized incident light, the absence of TE-TM interference gives rise to only six intensity peaks in this angular interval. For the size parameter used here, the supernumerary rainbow rings blend into the glory rings, rather than having separate rainbow and glory structures, as is the case for larger size spheres. According to Eq. (21), if Airy theory were quantitatively accurate all the way out to $180^{\circ}$, there should be five peaks in the interval $143.60^{\circ} \leq \Theta \leq 180.0^{\circ}$. The comparison is again good, and the fact that there are only a few peaks in the angular interval for the size 
parameter used here will be important later in our discussion of the interference structure of the transverse cusp and hufs.

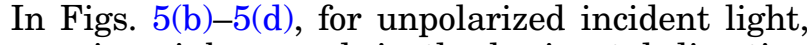
the main rainbow peak in the horizontal direction is observed to remain at a nearly constant angular position $\left(\Theta=143.60^{\circ}, 143.86^{\circ}\right.$, and $144.64^{\circ}$, respectively), while it is observed to noticeably shift in the vertical direction $\left(\Theta=147.60^{\circ}, 163.35^{\circ}\right.$, and $171.00^{\circ}$, respectively). Assuming that the Airy and Mobius $[40,41]$ corrections to the rainbow position are additive, the main rainbow peak in the vertical direction on the viewing screen for this size parameter should occur at $\Theta=148.39^{\circ}, 156.52^{\circ}$, and $163.35^{\circ}$ for $a / b=1.05,1.15$, and 1.25 , respectively. This prediction agrees well with Fig. 5(b), it underestimates the observed angle in Fig. 5(c), and it greatly underestimates the observed angle in Fig. 5(d). This is not surprising because the Mobius correction is only the first term of a series expansion of the Descartes rainbow angle in powers of $(1-b / a)$ and, as such, it is not expected to be quantitatively valid for $a / b$ as large as 1.25. Considering now Figs. 4(b)-4(d) for vertically polarized incident light, the most evident difference of these figures from Fig. 4(a) is that the scattered light near the vertical axis becomes increasingly dimmer due to either decreased refraction or total internal reflection at the $p=2$ interface, as was mentioned in [9]. In addition, according to Eq. (33) in the short wavelength limit, the transverse cusp should first form from real rays for $a / b=1.068$ at $\Theta_{\mathrm{cp}}=165.77^{\circ}$, although for $a / b$ somewhat smaller than this, such as in Fig. 5(b) for $a / b=1.05$, the beginnings of the transverse cusp can still weakly form due to the interference of surface waves rather than of real rays. From Eqs. (1) and (31) for $a / b=1.15$, the transverse cusp point should be located at $\Theta_{\mathrm{cp}}=152.06^{\circ}$. According to Eqs. (35) (37), however, the first interference peak on the horizontal axis inside the cusp caustic is predicted to be sufficiently wide that it cannot completely fit into the angular interval $\Theta_{\mathrm{cp}}<\Theta<180^{\circ}$ for this small $a / b$ ratio. In Figs. 4(c) and 5(c), for $a / b=1.15$, a prominent interference peak is observed on the horizontal axis at $\Theta=155^{\circ}$. But it is uncertain how much of it is attributable to the main peak of slowly growing transverse cusp and how much is attributable to the azimuthal contraction of the first supernumerary maximum of the rainbow to the $\Phi$ region centered on the horizontal axis, which, according to Eq. (21), should occur at $\Theta=156.09^{\circ}$.

For $a / b=1.25$, according to Eqs. (1) and (31), the transverse cusp point should now occur at $\Theta_{c p}=$ $140.58^{\circ}$ and, from Eqs. (35)-(37), the first interference peak on the horizontal axis inside the cusp should be located at $\Theta=152.73^{\circ}$. In Figs. 4(d) and $5(\mathrm{~d})$, for $a / b=1.25$, the first rainbow supernumerary maximum on the axis is absent and appears to have destructively interfered with this peak. The main rainbow peak on the horizontal axis is instead flanked by a new set of interference peaks slightly above and below the axis, at a scattering angle of $\Theta=152^{\circ}$. This suggests that the new peaks above and below the horizontal axis are associated with the beginnings of the formation of the first peak of the transverse cusp intensity above and below the caustic axis (see Fig. 1 of [37]). Because the interference structure of both the rainbow and transverse cusp are broad and substantially overlap for the spheroid size parameter used here, Figs. 4(c), 4(d), $5(\mathrm{c})$, and $5(\mathrm{~d})$ qualitatively support the case that the size parameter is too small to cleanly fit very much of the relatively wide interference structure of the transverse cusp into the relatively narrow angular interval between the cusp curves. This situation was also encountered in [43] for the asteroid caustic of spheroid glory scattering.

According to Eq. (34), the hyperbolic umbilic focal section should occur for $(a / b)_{\text {hufs }}=1.308$. Figures $4(\mathrm{e})$ and $5(\mathrm{e})$ show the scattered intensity for $a / b=1.30$, which is near the hufs condition. In Fig. 4(e), an interference pattern inside the caustic curve is now clearly evident. But it is uncertain from the figure whether the interference pattern is that of the transverse cusp or the hufs. To verify that $a / b=$ 1.30 is quite close to $(a / b)_{\text {hufs }}$ for the spheroid size parameter used here, we calculated the scattered intensity of the main peak on the horizontal axis for vertically polarized incident light for $1.28 \leq a / b \leq$ 1.33 in increments of $\Delta(a / b)=0.01$. Because the point of the hufs produces a higher degree of focusing than does the point of the transverse cusp, the relative maximum of the intensity's main peak should correspond to the hufs condition. This relative maximum was found to occur for $a / b=1.30$ and the intensity on the horizontal axis for this case is shown in Fig. 6. According to Eqs. (1), (31), (35), and (36), for $a / b \equiv 1.30$, the cusp caustic point should occur at $\Theta_{\text {cp }}=137.66^{\circ}$, the main peak should be displaced from it by $5.11^{\circ}$, and the second intensity peak on the axis should be displaced from the first peak by an additional $8.28^{\circ}$. But according to Eqs. (1) and (15), the hufs point should occur at the rainbow scattering angle $\Theta_{\text {hufs }}=137.48^{\circ}$, which is nearly the same as $\Theta_{\text {cp }}$. From Eq. (54), the first hufs intensity peak on the horizontal axis should be displaced from the caustic point by $9.04^{\circ}$, and the second hufs intensity peak should be displaced from the first peak by an additional $19.78^{\circ}$. In Fig. $\underline{6}$, the first and second angular displacements are $7.05^{\circ}$ and $11.48^{\circ}$, respectively, and are intermediate between these two predictions. This suggests that, as was the case for the transverse cusp for $a / b=1.15$ and 1.25 , the spheroid size parameter used here is again too small to cleanly fit all the features of the hufs diffraction pattern into the relatively narrow angular region between the branches of the caustic.

To further test this conjecture, we magnified the intensity graph in the region around the rainbow scattering angle for $a / b=1.30$ for vertically polarized incident light. This is shown in Fig. 7. The angle between the main diffraction peak on the horizontal 


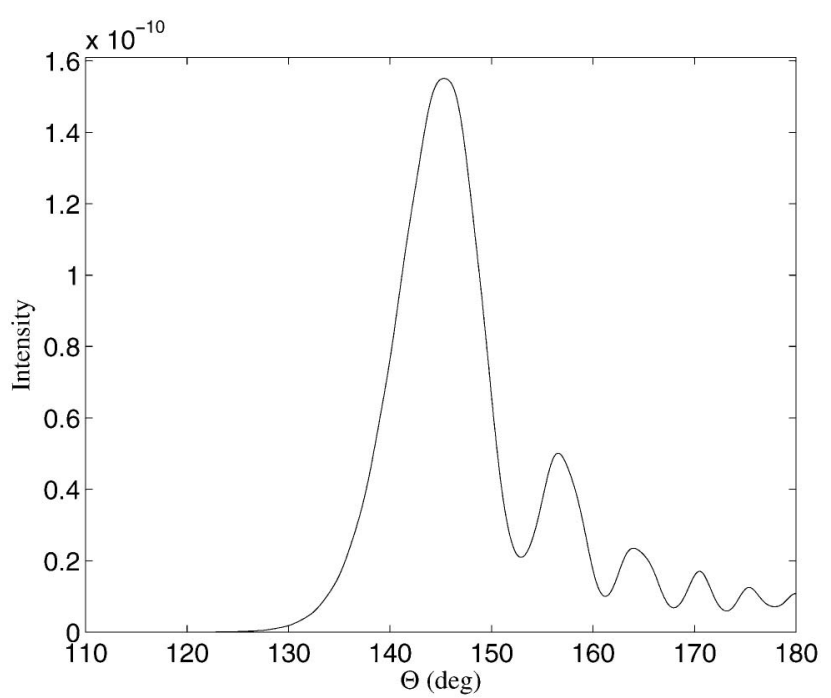

Fig. 6. Intensity as a function of the angle $\Theta$ along the horizontal axis of the exit plane for a vertically polarized incident plane wave with wavelength $\lambda=0.5145 \mu \mathrm{m}$ scattered by an oblate spheroid with refractive index $n=1.334+i\left(1.2 \times 10^{-9}\right)$, horizontal radius $a=6.0 \mu \mathrm{m}$, and radius ratio $a / b=1.30$.

axis and a number of off-axis peaks was measured and compared with the predictions of Eqs. (35)-(37) for the transverse cusp for this spheroid size parameter. We measured the angle $\xi=\arctan \left(\Delta_{V} / \Delta_{H}\right)$ between the horizontal axis and the line joining the

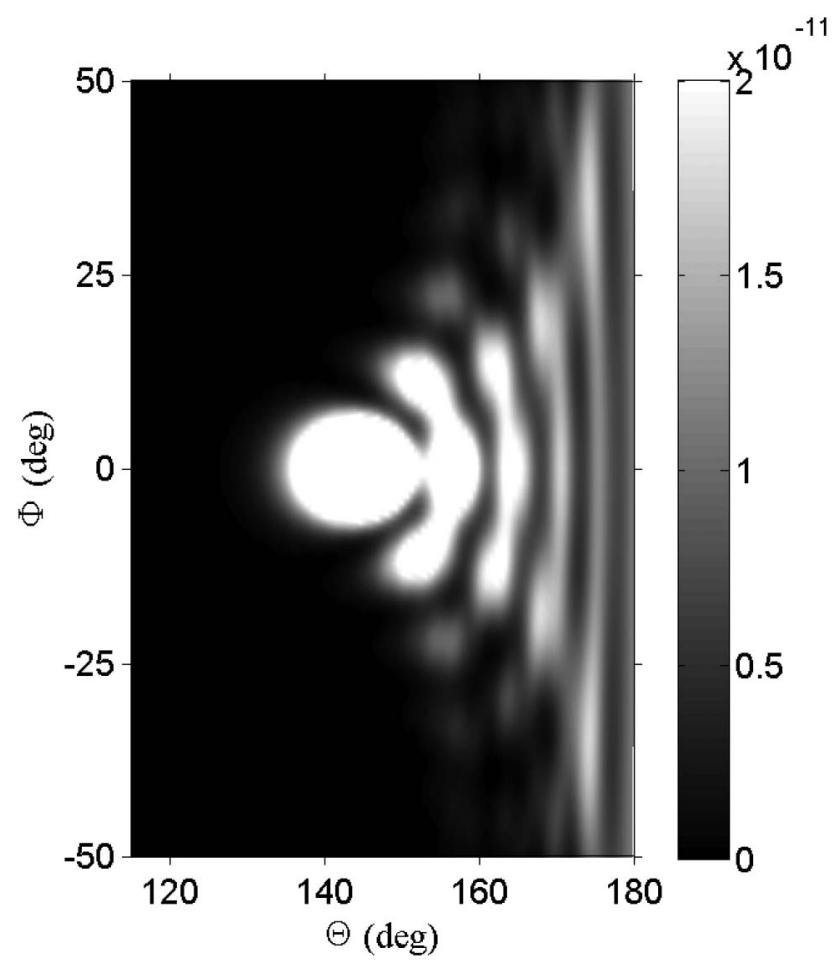

Fig. 7. Intensity as a function of the angles $\Theta$ and $\Phi$ or, equivalently, $\Delta_{H}$ and $\Delta_{V}$, on the far-zone viewing screen in the vicinity of the Descartes rainbow angle for a vertically polarized incident plane wave with wavelength $\lambda=0.5145 \mu \mathrm{m}$ scattered by an oblate spheroid with refractive index $n=1.334+i\left(1.2 \times 10^{-9}\right)$, horizontal radius $a=6.0 \mu \mathrm{m}$, and radius ratio $a / b=1.30$. first peak on the horizontal axis with each of the first three peaks in the first row of maxima parallel to the caustic curve (see Fig. 1 of [37]). Our results are $53^{\circ}$, $65^{\circ}$, and $70^{\circ}$, while the predictions of Eqs. (35)-(37) are $52.4^{\circ}, 54.9^{\circ}$, and $58.6^{\circ}$. The first peak is at almost exactly the correct position, while the second and third peaks are about 20\% larger than predicted. This strongly suggests that we are finally starting to see the interference structure of the transverse cusp caustic. According to Eqs. (54) and (55), if the caustic in Fig. 7 had been a hufs, the first peak on the horizontal axis and the first row of interference peaks parallel to the caustic curve would have all fallen on a straight line with $\xi=21.0^{\circ}$, which is very different than the angles observed in Fig. 7. The angle $\xi$ was also measured for the first two off-axis peaks in the second row of maxima parallel to the caustic curve. We obtained $\xi=35^{\circ}$ and $57^{\circ}$, whereas Eqs. (35)-(37) predict that they should occur at $\xi=$ $23.0^{\circ}$ and $33.6^{\circ}$. The percentage difference between the two sets of angles is now greatly increased, indicating that, even for $a / b=1.30$, the transverse cusp interference pattern inside the caustic curve has not yet fully formed. The reason for this is, again, the incompatibility between the large angular size of the peaks and the relatively small angular interval into which they must fit. Equations (9), (30), and (39), Figs. 4b-4f of [1], and Fig. 5 of [7] all indicate that the opening angle of the transverse cusp is rather small, except near the hufs condition, where it noticeably widens. Thus, if one wishes to resolve a number of interference peaks in the restricted angular region between the transverse cusp or hufs caustic curves, a large value of $k a$ at or near the hufs condition is required, because the angular shape of the caustic curve is independent of $k a$, whereas the angular size of the interference peaks decreases as a function of $k a$. Although a spheroid size parameter of $2 \pi a / \lambda \approx$ 73 is sufficient to exhibit the increased focusing at the point of the hufs, it is still too small to resolve the four-ray interference pattern inside the hufs.

Lastly, Eq. (33) predicts that, when $a / b=1.413$, the vertical portion of the rainbow caustic occurs at $\Theta=180^{\circ}$ and corresponds to the lips event. Figures 4(f) and 5(f) show the scattered intensity somewhat near this condition when $a / b=1.36$. The position of the main peak of the horizontal rainbow is $\Theta=144.25^{\circ}$, and has barely moved from where it was for $a / b=1.0001$. Attached to the horizontal rainbow is a weak cusplike structure. The remainder of the global caustic is the lips curve, a virtually straight line along the horizontal axis of the viewing screen that has a weak supernumerary structure at slightly smaller scattering angles in the vertical direction. This is contrasted with the case for $a / b=1.0001$, where the supernumerary structure in both the vertical and horizontal directions occurred for larger scattering angles.

In summary, although it has been long known that a number of different caustics are produced in scattering of a plane wave by a nonabsorbing oblate 
spheroid, this structure has, to the best of our knowledge, never been studied using exact electromagnetic scattering techniques, such as the extension of Mie theory to spheroid scattering. This paper has attempted to do so, and has also examined the features of the caustic structure that are and are not present when the spheroid is relatively small. We found that intensity enhancements are present at nearly the correct value of $a / b$, but that, for a clear and welldefined formation of more than the first few interference peaks between the two arms of the caustics, a larger size spheroid is required. As a last note, in analogy to the increasingly large size parameter of a sphere required to resolve the details of the rainbow for $p \geq 3$, the spheroid size parameter required to resolve the details of the transverse cusp caustics for $p \geq 3$ and the more complicated $p=2$ caustic progression at larger spheroid $a / b$ ratios is also likely to be larger than that required to resolve the details of the interference patterns of the $p=2$ caustics examined here.

This work was supported in part by the German Research Foundation (DFG) (grant TR194/49-1) and the Alexander von Humboldt Foundation (grant CHN/1127072).

\section{References and Note}

1. P. L. Marston and E. H. Trinh, "Hyperbolic umbilic diffraction catastrophe and rainbow scattering from spheroidal drops," Nature 312, 529-531 (1984).

2. J. F. Nye, "Rainbow scattering from spheroidal drops-an explanation of the hyperbolic umbilic foci," Nature 312, 531-532 (1984).

3. P. L. Marston, "Cusp diffraction catastrophe from spheroids: generalized rainbows and inverse scattering," Opt. Lett. 10, 588-590 (1985).

4. P. L. Marston, "Transverse cusp diffraction catastrophes: Some pertinent wave fronts and a Pearcey approximation to the wave field," J. Acoust. Soc. Am. 81, 226-232 (1987).

5. P. L. Marston, Erratum, J. Acoust. Soc. Am. 83, 1976 (1988)

6. P. L. Marston, C. E. Dean, and H. J. Simpson, "Light scattering from spheroidal drops: Exploring optical catastrophes and generalized rainbows," in Drops and Bubbles: American Institute of Physics Conference Proceedings 197, T. G. Wang, ed. (American Institute of Physics, 1989), pp 275-285.

7. C. E. Dean and P. L. Marston, "Opening rate of transverse cusp diffraction catastrophe in light scattering by oblate spheroidal drops," Appl. Opt. 30, 3443-3451 (1991).

8. C. E. Dean and P. L. Marston, Errata, Appl. Opt. 32, 2163 (1993). Also as a correction to [7], please replace $d_{\infty}$ in Eq. (6a) with $1 / d_{\infty}$.

9. J. F. Nye, "Rainbows from ellipsoidal water droplets," Proc. R. Soc. London Ser. A 438, 397-417 (1992).

10. S. Holler, Y. Pan, R. K. Chang, J. R. Bottiger, S. C. Hill, and D. B. Hillis, "Two-dimensional angular optical scattering for the characterization of airborne microparticles," Opt. Lett. 23, 1489-1491 (1998).

11. S. Asano and G. Yamamoto, "Light scattering by a spheroidal particle," Appl. Opt. 14, 29-49 (1975).

12. S. Asano and G. Yamamoto, Erratum, Appl. Opt. 15, 2028 (1976)

13. S. Asano, "Light scattering properties of spheroidal particles," Appl. Opt. 18, 712-723 (1979).
14. S. Asano and M. Sato, "Light scattering by randomly oriented spheroidal particles," Appl. Opt. 19, 962-974 (1980).

15. M. V. Berry, "Waves and Thom's theorem," Adv. Phys. 25, 1-26 (1976).

16. M. V. Berry and C. Upstill, "Catastrophe optics: morphologies of caustics and their diffraction patterns," Prog. Opt. 18, 257346 (1980).

17. H. C. van de Hulst, Light Scattering by Small Particles (Dover, 1981), Section 13.24.

18. F. Xu, J. A. Lock, and C. Tropea, "Debye series for light scattering by a spheroid," J. Opt. Soc. Am. A (in press).

19. Y. P. Han, L. Mees, K. F. Ren, G. Gouesbet, S. Z. Wu, and G. Grehan, "Scattering of light by spheroids: the far field case," Opt. Commun. 210, 1-9 (2002).

20. G. Kaduchak and P. L. Marston, "Hyperbolic umbilic and $E_{6}$ diffraction catastrophes associated with the secondary rainbow of oblate water drops: observations with laser illumination," Appl. Opt. 33, 4697-4701 (1994).

21. P. L. Marston and G. Kaduchak, "Generalized rainbows and unfolded glories of oblate drops: organization for multiple internal reflections and extension of cusps into Alexander's dark band," Appl. Opt. 33, 4702-4713 (1994).

22. D. S. Langley and P. L. Marston, "Generalized tertiary rainbow of slightly oblate drops: observations with laser illumination," Appl. Opt. 37, 1520-1526 (1998).

23. H. J. Simpson and P. L. Marston, "Scattering of white light from levitated oblate water drops near rainbows and other diffraction catastrophes," Appl. Opt. 30, 3468-3473, 3547 (1991).

24. G. Kaduchak, P. L. Marston, and H. J. Simpson, " $E_{6}$ diffraction catastrophe of the primary rainbow of oblate water drops: observations with white light and laser illumination," Appl. Opt. 33, 4691-4696 (1994).

25. J. W. Goodman, Introduction to Fourier Optics (McGraw-Hill, 1968), Chap. 5.

26. W. J. Humphreys, Physics of the Air (Dover, 1964), pp. $483-491$.

27. J. A. Adam, "The mathematical physics of rainbows and glories," Phys. Rep. 356, 229-365 (2002), Sections 1.2, 2.1.

28. H. C. van de Hulst, Light Scattering by Small Particles (Dover, 1981), Section 13.23

29. H. M. Nussenzveig, Diffraction Effects in Semiclassical Scattering (Cambridge U. Press, 1992), Section 3.2.

30. P. L. Marston, "Geometrical and catastrophe optics methods in scattering," Phys. Acoust. 21, 1-234 (1992), Section 2.3.

31. P. L. Marston, "Geometrical and catastrophe optics methods in scattering," Phys. Acoust. 21, 1-234 (1992), Fig. 7.

32. R. T. Wang and H. C. van de Hulst, "Rainbows: Mie computations and the Airy approximation," Appl. Opt. 30, 106-117 (1991).

33. H. M. Nussenzveig, "The theory of the rainbow," Sci. Am. 236 (4), 116-127 (1977).

34. J. A. Lock and E. A. Hovenac, "Internal caustic structure of illuminated liquid droplets," J. Opt. Soc. Am. A8, 15411552 (1991).

35. M. Abramowitz and I. A. Stegun, Handbook of Mathematical Functions (National Bureau of Standards, 1964), Eq. 10.4.32.

36. M. Abramowitz and I. A. Stegun, Handbook of Mathematical Functions (National Bureau of Standards, 1964), Eqs. 10.4.59, 10.4.60.

37. T. Pearcey, "The structure of an electromagnetic field in the neighbourhood of a caustic," Philos. Mag. 37, 311-317 (1946).

38. J. N. L. Connor and D. Farrelly, "Theory of cusped rainbows in elastic scattering: uniform semiclassical calculations using Pearcey's integral," J. Chem. Phys. 75, 2831-2846 (1981). 
39. M. V. Berry, J. F. Nye, and F. J. Wright, "The elliptic umbilic diffraction catastrophe," Phil. Trans. R. Soc. London 291, 453-484 (1979), Fig. 13.

40. W. Mobius, "Zur Theorie des Regenbogens und ihrer experimentallen Prufung," Abh. Kgl. Saechs. Ges. Wiss. Math.-Phys. Kl. 30, 105-254 (1907-1909).

41. W. Mobius, "Zur Theorie des Regenbogens und ihrir experimentallen Prufung," Ann. Phys. (Leipzig) 33, 1493-1558 (1910).

42. G. P. Konnen and J. H. de Boer, "Polarized rainbow," Appl. Opt. 18, 1961-1965 (1979).

43. W. P. Arnott and P. L. Marston, "Unfolding axial caustics of glory scattering with harmonic angular perturbations of toroidal wave fronts,” J. Acoust. Soc. Am. 85, 1427-1440 (1989).
44. W. P. Arnott and P. L. Marston, "Unfolded optical glory of spheroids: Backscattering of laser light from freely rising spheroidal air bubbles in water," Appl. Opt. 30, 3429-3442 (1991).

45. A. Thaning, Z. Jaroszewicz, and A. T. Friberg, "Diffractive axicons in oblique illumination: analysis and experiments and comparison with elliptical axicons," Appl. Opt. 42, 9-17 (2003).

46. H. M. Nussenzveig, "High-frequency scattering by a transparent sphere. II. Theory of the rainbow and the glory," J. Math. Phys. 10, 125-176 (1969).

47. M. V. Berry and C. Upstill, "Catastrophe optics: morphologies of caustics and their diffraction patterns," Prog. Opt. 18, 257346 (1980), Appendix 2. 